\title{
Riqueza de especies de invertebrados en playas de arena y costas rocosas del Pacífico Norte de Costa Rica
}

\author{
Jeffrey Alejandro Sibaja-Cordero ${ }^{1,2}$, Yolanda E. Camacho-García ${ }^{1,2}$ \& Rita Vargas-Castillo ${ }^{1,2}$ \\ 1. Centro de Investigación en Ciencias del Mar y Limnología (CIMAR), Universidad de Costa Rica, San Pedro, 11501- \\ 2060 San José, Costa Rica; jeffrey.sibaja@ucr.ac.cr \\ 2. Escuela de Biología \& Museo de Zoología, Universidad de Costa Rica, San Pedro, 11501-2060 San José, Costa Rica
}

Recibido 22-X-2013. Corregido 10-IV-2014. Aceptado 27-IX-2014.

\begin{abstract}
Invertebrate species richness in sandy beaches and rocky coast of Costa Rican North Pacific. This study summarizes published information on the number of invertebrate species reported for the intertidal zone of rocky coasts and sandy beaches of the North Pacific of Costa Rica. In addition, we present new data on macrofaunal species richness for several locations. There is a wide variation in the number of species reported, with mollusks, crustaceans, and polychaetes as the major contributors. The number of species depends on the faunal group and the sampled effort carried out in the previous studies of the intertidal zones, and during 2012. The data show that, taking into account all components of the community values, a range of 29-172 species were obtained from the rocky intertidal, and 5-15 species for the sandy beaches. We found a similar mean value for species richness between protected and not protected areas, because in both cases the range of values is wide. It is urgent to manage unprotected locations with high species richness that are exposed to impacts from development of human populations. Rev. Biol. Trop. 62 (Suppl. 4): 63-84. Epub 2014 Diciembre 01.
\end{abstract}

Key words: Species richness, tropical beaches, Crustacea, Mollusca, Polychaeta, algae.

La franja litoral que contiene las playas de arena y costas rocosas ha sido fuertemente impactada por el desarrollo humano en diferentes lugares alrededor del mundo. En paises tropicales, en los cuales gran parte de su economía está basada en las actividades turísticas, el desarrollo de infraestructuras y el mal manejo del uso del suelo pueden provocar impactos sobre dichos hábitats, dañando la fauna y flora marina (Defeo et al., 2009). En Costa Rica, la zona del Pacífico Norte cuenta tanto con áreas con un gran desarrollo de poblaciones humanas asociadas al turismo de playa; así como con zonas protegidas por el Ministerio de Ambiente y Energía, y que van desde Refugios de Vida Silvestre y Parques Nacionales hasta una Reserva Absoluta.

Para el Pacífico Norte de Costa Rica se cuenta con pocos trabajos publicados sobre la biota de las zonas intermareales. Listados de taxones en trabajos ecológicos se pueden encontrar para las zonas rocosas de Playas del Coco (Spight, 1978), Bahía Ballena y Montezuma (Villalobos, 1980a; b; Sibaja-Cordero \& Vargas-Zamora, 2006), Bahía Culebra (Madrigal-Castro et al., 1984) y Bahía Salinas (SibajaCordero \& Cortés, 2008). Solamente hay un único trabajo realizado sobre las comunidades biológicas presentes en las playas arenosas (Dexter, 1974) correspondientes a playas del Coco, Tamarindo y Sámara. También, existen listas y estudios taxonómicos, sobre poliquetos (Dean, 2004; 2009) y sipuncúlidos (Dean, 2001b; Vargas \& Dean, 2009), trabajos en los que se especifican los sitios de recolecta y si la especie ha sido encontrada en zona intermareal.

El objetivo de este trabajo es hacer una revisión de la biota en la región del Pacífico 
Norte mediante un sondeo de la diversidad biológica y con información disponible sobre invertebrados de las playas arenosas y zonas rocosas. Esto incluye riqueza de taxones, abundancia relativa y distribución a partir de datos publicados o mediante giras de evaluación y recolección de especímenes, en zonas con y sin medidas de conservación.

\section{MATERIALES Y MÉTODOS}

Se estimó la riqueza de taxones de invertebrados marinos, su distribución y abundancia relativa en las comunidades intermareales (playas arenosas y costas rocosas) del Pacífico Norte de Costa Rica. Para dicha región, se revisó la literatura existente sobre zonas intermareales y se realizaron visitas a localidades que no habían sido estudiadas previamente. Para la toma de muestras y datos de presencia y distribución de los invertebrados se muestrearon las zonas intermareales durante la marea baja, para la recolecta de especímenes, datos de presencia y distribución de los invertebrados. Estos puntos de muestreo fueron, de norte a sur de la Costa Pacífica:

- Sector Santa Elena:

- El Hachal, Sector Murciélago, Golfo de Santa Elena, zona rocosa, visitada el 31/05/2012.

- Junquillal, Sector Murciélago, Bahía Junquillal, zona rocosa y playa de arena, visitada el 31/06/2012.

- Sector Golfo de Papagayo:

- Hermosa, zona rocosa y playa de arena, visitada el 07/02/2012.

- Matapalo, zona rocosa y playa de arena, visitada el 08/02/2012.

- Sector Punta Gorda-Punta Pargos:

- Carbón, Parque Nacional Marino las Baulas, zona rocosa, visitada el 10/02/2012.

- Ventanas, Parque Nacional Marino las Baulas, zona rocosa y playa de arena, visitada el 09/02/2012.

- Sector Cabo Blanco:
- San Miguel, Reserva Absoluta Cabo Blanco, zona rocosa, visitada el 21 y 22/02/2012.

- Cabuya, Reserva Absoluta Cabo Blanco, zona rocosa, visitada el 23/02/2012.

- Tambor, zona rocosa y playa de arena, visitada el 24/02/2012.

Se anotaron los taxones presentes y se estimó su abundancia o cobertura relativa en cada estrato de los transectos para las playas que se visitaron. Todos los organismos recolectados se identificaron hasta el nivel taxonómico más bajo posible utilizando guías especializadas, y se estimó su abundancia o cobertura relativa en cada estrato de los transectos de las localidades visitadas. Los moluscos se identificaron mediante los trabajos de Keen (1971), Fischer et al. (1995) y Camacho-García, Gosliner \& Valdés (2005), los cirripedios con Laguna (1985), los poliquetos con de León et al. (2009) y para los crustáceos se usaron: Rathbun (1930), Garth (1958), Haig (1960), Kim \& Abele (1988), Fischer et al. (1995), Hendrickx (1999) y Salgado-Barragan \& Hendrickx (2010), entre otras guías. Además, para ambos ambientes, se buscó la información publicada en revistas científicas y se actualizó el número de taxones presentes en cada localidad. Para cada una se da una descripción de la costa rocosa o playa de arena y del tipo de substrato algal dominante, utilizando para este último la guía de Fischer et al. (1995).

\section{Costa rocosa}

Para cuantificar en el caso de la epifauna presente, tanto organismos móviles como sésiles, se ubicaron cinco cuadrículas de 25 por $25 \mathrm{~cm}$ en cada uno de los cinco estratos de la zona intermareal de cada localidad (supralitoral, litoral alto, medio y bajo, e infralitoral), (Sibaja-Cordero \& Vargas-Zamora, 2006). Los grupos de las costas rocosas intermareales ocupan una gran variedad de microhábitats, y muchos son difíciles de observar porque se encuentran dentro o bajo las rocas, y entre las algas. El trabajo para su recolecta resulta difícil 
y para los grupos móviles y endolíticos debe hacerse dirigido y no al azar o sistemático, por lo que se realizó un recorrido por las costas rocosas buscando debajo de las rocas, en grietas y pozas intermareales. Se recolectaron algas manualmente y por raspado de la roca para permitir a su vez la recolecta de la fauna encontrada, incluyendo posteriormente la técnica del remonte (Fischer, 1981; Camacho-García et al., 2005).

Inmediatamente después del muestreo, los organismos fueron separados del sustrato utilizando el estereomicroscopio. En algunos casos fue necesario relajar a los individuos utilizando cloruro de magnesio disuelto en agua de mar. Adicionalmente, se documentó la coloración de algunos especímenes mediante la toma de fotografías in vivo. Finalmente, el material fue clasificado a la menor categoría taxonómica posible en ese momento durante la gira, y aquellos organismos que requerían de un estudio más detallado de sus características anatómicas fueron trasladados al laboratorio (CIMAR o Museo de Zoología de la Universidad de Costa Rica).

\section{Playas de arena}

Para este hábitat, se utilizó el muestreo por medio de cilindros con un área de $18 \mathrm{~cm}^{2}$ que fueron enterrados hasta $15 \mathrm{~cm}$ en el sedimento. Así, se tomaron al menos tres muestras de sedimento de forma sistemática en cada estrato del intermareal: supralitoral, litoral alto, medio y bajo, e infralitoral (Dexter, 1974). Cada muestra fue tamizada para retener los organismos superiores a 500 micras (Dexter, 1974; Vargas, 1987). Se realizó un recorrido explorando con una cuadrícula circular de $56.5 \mathrm{~cm}$ de radio ( $1 \mathrm{~m}^{2}$ de área) a $15 \mathrm{~cm}$ de profundidad en el sedimento en cada uno de los cinco estratos para determinar la presencia de otros grupos de macrofauna. Las muestras se analizaron y contabilizaron durante la gira o en el laboratorio del CIMAR utilizando el estereomicroscopio. Estos se preservaronen alcohol al $95 \%$ y clasificándos e identificaron de igual manera que en el caso anterior.
Se comparó el promedio para todos los datos recolectados (literatura y campo) de las zonas rocosas intermareales; pero también, se realizó la comparación sólo para las localidades muestreadas en el presente estudio, $\mathrm{y}$, finalmente se comparó la información generada en este trabajo para las playas de arena con los datos de Dexter (1974). Dichas comparaciones fueron realizadas mediante una $t$ de dos muestras, comprobando la normalidad de residuos e igualdad de varianza en los datos. Los datos fueron representados geográficamente y temporalmente para evaluar la posible existencia de tendencias.

\section{RESULTADOS}

\section{Costa rocosa}

La riqueza de especies encontrada en las costas rocosas estudiadas durante 2012 se puede apreciar en el Cuadro 1. Para algunos grupos taxonómicos; es de destacar que lugares protegidos como Cabuya, Playa El Hachal y Playa Carbón representaron puntos de baja riqueza de especies para algunos grupos taxonómicos.

\section{Sector Santa Elena:}

El Hachal: La zona intermareal rocosa presentó cantos rodados de gran tamaño. Esta localidad cuenta con medidas de conservación, ya que pertenece al Área de Conservación Guanacaste (ACG). Esta ubicada a unos $2 \mathrm{~km}$ del poblado más cercano. No se observaron parches de macroalgas; se detectó solamente una delgada película turf compuesta por algas verdes y cianobacterias que recubría los cantos en la zona más baja. Solo un número de cinco especies de invertebrados fue observado debajo de los bloques o de cantos rodados.

Crustáceos: La riqueza presentó valores más bajos en cuanto al número de familias (8) y únicamente once especies se hallaron (Cuadro 1). Las únicas especies abundantes fueron el cangrejo guisante Glassella costaricana (Wicksten, 1982), que vive asociada al 
CUADRO 1

Riqueza de especies según categoría taxonómica de crustáceos, moluscos, poliquetos y otros invertebrados en las costas rocosas del Pacífico Norte de Costa Rica evaluadas en el presente estudio. n.d.= no hay datos disponibles. ZP: Zona protegida por el Ministerio de Ambiente, NP: Zona no protegida. Las localidades están ordenadas de Norte a Sur

\begin{tabular}{|c|c|c|c|c|c|}
\hline Sitio & Grupo taxonómico & Clase & Familia & Género & Especie \\
\hline El Hachal & Crustacea & 1 & 8 & 8 & 11 \\
\hline $10^{\circ} 56.128^{\prime} \mathrm{N}-85^{\circ} 43.725^{\prime} \mathrm{W}$ & Mollusca & 3 & 14 & 17 & 18 \\
\hline \multirow[t]{2}{*}{$\mathrm{ZP}$} & Polychaeta & 1 & 1 & 1 & 1 \\
\hline & Otros & 0 & 0 & 0 & 0 \\
\hline Junquillal & Crustacea & 1 & 15 & 20 & 23 \\
\hline $10^{\circ} 57.803^{\prime} \mathrm{N}-85^{\circ} 41.571^{\prime} \mathrm{W}$ & Mollusca & 2 & 19 & 28 & 29 \\
\hline \multirow[t]{2}{*}{$\mathrm{ZP}$} & Polychaeta & 1 & 7 & 7 & 9 \\
\hline & Otros & 5 & 3 & 4 & 7 \\
\hline Virador & Crustacea & 2 & 1 & 3 & 4 \\
\hline $10^{\circ} 36.734^{\prime} \mathrm{N}-85^{\circ} 41.443^{\prime} \mathrm{W}$ & Mollusca & 2 & 7 & 9 & 10 \\
\hline \multirow[t]{2}{*}{ NP } & Polychaeta & n.d. & n.d. & n.d. & n.d. \\
\hline & Otros & 1 & 0 & 0 & 1 \\
\hline Hermosa & Crustacea & 3 & 19 & 33 & 41 \\
\hline $10^{\circ} 34.227^{\prime} \mathrm{N}-85^{\circ} 41.083^{\prime} \mathrm{W}$ & Mollusca & 2 & 30 & 52 & 61 \\
\hline \multirow[t]{2}{*}{ NP } & Polychaeta & 1 & 14 & 14 & 20 \\
\hline & Otros & 6 & 5 & 5 & 8 \\
\hline Matapalo & Crustacea & 3 & 30 & 39 & 56 \\
\hline $10^{\circ} 32.028^{\prime} \mathrm{N}-85^{\circ} 44.763^{\prime} \mathrm{W}$ & Mollusca & 3 & 43 & 66 & 80 \\
\hline \multirow[t]{2}{*}{ NP } & Polychaeta & 1 & 7 & 7 & 12 \\
\hline & Otros & 3 & 3 & 4 & 6 \\
\hline Carbón & Crustacea & 2 & 22 & 25 & 30 \\
\hline $10^{\circ} 20.782^{\prime} \mathrm{N}-85^{\circ} 51.693^{\prime} \mathrm{W}$ & Mollusca & 2 & 8 & 9 & 9 \\
\hline \multirow[t]{2}{*}{$\mathrm{ZP}$} & Polychaeta & 1 & 5 & 6 & 8 \\
\hline & Otros & 3 & 3 & 3 & 3 \\
\hline Ventanas, & Crustacea & 2 & 13 & 20 & 21 \\
\hline $10^{\circ} 20.714^{\prime} \mathrm{N}-85^{\circ} 51.612^{\prime} \mathrm{W}$ & Mollusca & 2 & 32 & 42 & 51 \\
\hline \multirow[t]{2}{*}{$\mathrm{ZP}$} & Polychaeta & 1 & 4 & 4 & 4 \\
\hline & Otros & 2 & 2 & 2 & 4 \\
\hline Sámara & Crustacea & 1 & 9 & 13 & 15 \\
\hline $9^{\circ} 52.277>\mathrm{N}-85^{\circ} 31.592^{\prime} \mathrm{W}$ & Mollusca & 2 & 33 & 48 & 65 \\
\hline \multirow[t]{2}{*}{$\mathrm{NP}$} & Polychaeta & n.d. & n.d. & n.d. & n.d. \\
\hline & Otros & n.d. & n.d. & n.d. & n.d. \\
\hline San Miguel (Reserva Cabo Blanco) & Crustacea & 3 & 21 & 34 & 40 \\
\hline $9^{\circ} 34.773^{\prime} \mathrm{N}-85^{\circ} 8.274^{\prime} \mathrm{W}$ & Mollusca & 2 & 50 & 70 & 96 \\
\hline \multirow[t]{2}{*}{$\mathrm{ZP}$} & Polychaeta & 1 & 14 & 14 & 30 \\
\hline & Otros & 5 & 5 & 6 & 9 \\
\hline Cabuya (Reserva Cabo Blanco) & Crustacea & 2 & 9 & 15 & 20 \\
\hline $9^{\circ} 35.297^{\prime} \mathrm{N}-85^{\circ} 5.291^{\prime} \mathrm{W}$ & Mollusca & 2 & 12 & 13 & 15 \\
\hline \multirow{2}{*}{$\mathrm{ZP}$} & Polychaeta & 1 & 13 & 17 & 30 \\
\hline & Otros & 2 & 2 & 2 & 6 \\
\hline Tambor & Crustacea & 3 & 18 & 25 & 34 \\
\hline $9^{\circ} 42.792^{\prime} \mathrm{N}-85^{\circ} 0.758^{\prime} \mathrm{W}$ & Mollusca & 2 & 11 & 14 & 15 \\
\hline \multirow[t]{2}{*}{$\mathrm{NP}$} & Polychaeta & 0 & 0 & 0 & 0 \\
\hline & Otros & 0 & 0 & 0 & 0 \\
\hline
\end{tabular}

poliqueto terebélido Lanicola guillermoi Capa \& Hutchings, 2006, y además, camarones del género Alpheus, fueron comunes en el litoral bajo.

Moluscos: El tipo de substrato rocoso hace que se dificulte el establecimiento de poblaciones salvo algunas especies de gasterópodos como Vasula melones (Duclos, 1832) y lapas. En total se encontraron 18 especies (Cuadro 1).

Poliquetos: La única especie presente fue L. guillermoi, que fabrica tubos con moco y arena gruesa debajo de las rocas.

Otros: La zona no mostraba otros grupos de invertebrados durante el muestreo. 
Junquillal: Esta Plataforma intermareal rocosa cuenta con cantos rodados. La localidad es zona protegida, del Sector Junquillal del ACG, a $2 \mathrm{~km}$ del poblado más cercano. Se encontró una cobertura alta de algas rojas frondosas y baja de algas rojas incrustantes calcáreas en el infralitoral y litoral bajo; las primeras presentaron una cobertura media y las últimas desaparecieron en la zona media del litoral. En dicha zona, solo aparecieron algas pardas incrustantes así como baja cobertura de algas del género Bostrychia. Algas verdes del género Ulva se encontraron en el infralitoral mostrando una cobertura media.

Crustáceos: Se encontraron 23 especies (Cuadro 1). Los cirripedios Balanus inexpectatus Pilsbry, 1916, mostraron cobertura baja en el infralitoral y litoral bajo, mientras que el litoral medio y alto presentó cobertura media y alta de Chthamalus panamensis Pilsbry, 1916, y baja de Tetraclita stalactifera (Lamarck, 1818). En el supralitoral se encontraron parches aislados de C. panamensis.

Moluscos: Se encontraron 29 especies (Cuadro 1). Familias bien representadas en términos de abundancia fueron Muricidae y Columbellidae. El infralitoral se presentó cobertura alta de los bivalvos Chama echinata Broderip, 1835, y Brachidontes sp. Fue notorio encontrar grandes colonias vivas de la ostra Saccostrea palmula (Carpenter, 1857), debido a que se trata de una zona protegida y la especie no presenta depredación por humanos. Los caracoles de la familia Littorinidae presentaron mayores densidades en el litoral alto y menores en el supralitoral; las lapas pulmonadas del género Siphonaria aparecieron en baja densidad.

Poliquetos: En el infralitoral la cobertura por colonias de Sabellariidae fue muy baja. Aparecieron, asimismo, familias de hábitos depredadores como Glyceridae, Lumbrineridae, Nereididae, y suspensívoros y depositívoros como Chaetopteridae, Pectinariidae y Terebellidae, constituyendo en su conjunto un total de nueve taxones (Cuadro 1).

Otros: Se encontraron los sipuncúlidos Antillesoma antillarum (Grube, 1858),
Phascolosoma sp., en las grietas de la roca y Sipunculus nudus Linnaeus, 1766, en sedimentos. En la roca fueron hallados nemertinos, el erizo Echinometra vanbrunti A. Agassiz, 1863, pepinos de mar (Holothuroidea) y anémonas.

\section{Sector Golfo de Papagayo:}

Hermosa: Plataforma de basalto intermareal con sectores de cantos rodados, resguardada del oleaje fuerte. No es zona protegida y está situada en las inmediaciones del poblado, por lo que cuenta con infraestructura tras la playa que puede ser una fuente de alteración del hábitat, aportando sedimentación y presiones sobre el sistema (pescadores de moluscos y turistas). $\mathrm{El}$ infralitoral estaba cubierto casi enteramente por Caulerpa sertularioides (S.G. Gmelin) M.A. Howe, 1905, que desplaza a los otros tipos algales y siendo la cobertura de algas rojas incrustantes y frondosas muy baja. En el nivel litoral se dieron algas verdes frondosas en muy baja cobertura (ej. incluyendo, parches del género Ulva).

Crustáceos: Los crustáceos sumaron un total de 41 especies (Cuadro 1). En dicha zona rocosa se dio una mezcla de cinco especies de cirripedios, todas ellas con coberturas menores al 25\%. En el infralitoral se encontró Megabalanus coccopoma (Darwin, 1854). En el litoral medio y alto se dieron coberturas sobre el 50\% de C. panamensis, y en el supralitoral se encontró Euraphia rhizophorae (Oliveira, 1940) en pequeños parches.

Moluscos: En esta playa se contó con un total de 61 especies, siendo los gasterópodos los más abundantes (Cuadro 1). Se encontraron bivalvos de importancia comercial tales como Pinna rugosa G.B. Sowerby I, 1835, Pinctada mazatlanica (Hanley, 1856) y S. palmula. De este último bivalvo se observó que muchos especímenes de al menos $10 \mathrm{~cm}$ de longitud habían sido depredados posiblemente en los últimos años; en esta playa, además, había poca cobertura de bivalvos vivos de la familia Ostreidae, pues solo se hallaba la valva cementada a la roca. Las familias Columbellidae, Neritidae y Littorinidae son las que presentaron 
el mayor número de individuos en el área. La zona supralitoral tiene una densidad alta del gastrópodo Echinolittorina modesta (Philippi, 1846). Se recolectaron dos especímenes de la familia Runcinidae, constituyendo el segundo registro en el Pacífico costarricense de una especie no descrita para la ciencia.

Poliquetos: Un total de 20 especies aparecieron en esta zona rocosa (Cuadro 1), siendo dominantes en las grietas y entre las algas las familias de poliquetos Eunicidae y Nereididae. Dichas familias cuentan con dos o más especies, siendo comunes los géneros Neanthes, Nereis, Eunice y Lysidice, entre otros. Además, se hallaron colonias poco desarrolladas del Sabellariidae Phragmatopoma (Familia Sabellariidae).

Otros: Se divisaron pocas esponjas y otros invertebrados bajo las rocas. Se encontró al erizo E. vanbrunti, varias anémonas, nemertinos y sipuncúlidos. En total se encontraron ocho especies (Cuadro 1).

Matapalo: La zona intermareal es una plataforma rocosa parte de un acantilado de basalto con pozas intermareales, y una pequeña zona de cantos rodados. La zona no cuenta con medidas de protección y es contigua a un desarrollo turístico de gran escala, lo que puede ser una fuente de alteración del hábitat, ya sea directa o indirectamente, al existir aporte de sedimentación (nueva infraestructura) y presión sobre el sistema (pescadores de moluscos o turistas). Aparecienron en el infralitoral algas rodófitas calcáreas incrustantes y frondosas, entre ellas, una con alta cobertura $(30 \%)$ del género Amphiroa. También, apareció el alga verde $C$. sertularioides con alrededor de un $50 \%$ de cobertura. En la zona litoral la cobertura es menor, salvo por las formas algales incrustantes, pero se dieron pequeños parches de algas rojas frondosas, y otras especies de algas verdes.

Crustáceos: Aparecen aquí los mayores números de familias, géneros y especies encontrados $(30,39$ y 56 respectivamente, Cuadro 1), a pesar de la cercanía con la infraestructura hotelera. Se encontró el cirripedio $T$. stalactifera por debajo de la banda de C. panamensis. Otro cirripedio (aún no identificado) fue hallado en el litoral alto y E. rhizophorae en el supralitoral.

Moluscos: Se identificaron 80 especies (Cuadro 1); los gasterópodos fueron los más abundantes. En el infralitoral se presentó los bivalvos $C$. echinata con baja cobertura, mientras en el litoral medio aparece $S$. palmula en densidad media. En el supralitoral se dan caracoles de la familia Littorinidae, como $E$. modesta en alta densidad, y los burgados (Neritidae) con poblaciones pequeñas.

Se observaron varios parches de la lapa con hueco, Fissurella virescens G.B. Sowerby, 1835 y del caracol depredador Planaxis planicostatus G.B. Sowerby I, 1825, en pequeñas pozas de mareas. Aparecieron muy pocos individuos, y de pequeño tamaño, de la lapa pulmonada del género Siphonaria. Tanto Siphonaria como el bivalvo Crassostrea son explotados ocasionalmente en la zona. También, se encontró una nueva especie de gasterópodo sacogloso, asociado al alga verde Halimeda sp. Aunque esta especie no ha sido descrita todavía ya se ha recolectado en otras localidades del Pacífico de Costa Rica.

Poliquetos: Aparecieron en total 12 especies (Cuadro 1) siendo abundantes las familias Eunicidae y Nereididae, con representantes de los géneros Neanthes, Nereis y Eunice. Asimismo fueron halladas otras familias, como los tubícolas Terebellidae, y los errantes Polynoidae. Las colonias de Sabellariidae se encontraron poco desarrolladas y varias estaban sumamente deterioradas, apareciendo marcas indicativas de que existieron en el pasado.

Otros: Se encontró muy pocos invertebrados por debajo de las rocas, un ejemplo fueron los erizos Hesperocidaris asteriscus H.L. Clark, 1948 y E. vanbrunti, además de un nemertino, anémonas y los sipuncúlidos $A$. antillarum y Phascolosoma sp.

\section{Sector Punta Gorda-Punta Pargos:}

Ventanas y Carbón: Localidades protegidas dentro del Parque Nacional Marino Las 
Baulas. En ambas, la zona intermareal es una plataforma rocosa de basaltos de poca pendiente, con muchos cantos rodados y del tipo más acantilado en playa Carbón. En esta última localidad en la parte superior se presentó un supralitoral de arena negra y roca del tipo toba volcánica. Estos intermareales están ubicados respectivamente a 300 y $500 \mathrm{~m}$ del poblado más cercano. Se presentó gran variedad de substrato algal en el infralitoral, con los géneros Halimeda, Dyctiota, Padina, Amphiroa y Codium con cobertura de media a alta (30 a $50 \%$ ) en ese estrato, y poca dominancia de algas incrustantes. En la zona litoral solo se dieron algas verdes frondosas en muy baja cobertura (10\%).

Crustáceos: En playa Carbón se encontraron 30 especies y en Ventanas 21 (Cuadro 1), siendo en general las poblaciones muy ricas y abundantes. En el caso de los cirripedios, en Ventanas se encontró $T$. stalactifera con muy baja cobertura, siempre ubicados debajo de la banda de $C$. panamensis. Apareció además, en el supralitoral de Ventanas, E. rhizophorae, estando ausente en playa Carbón.

Moluscos: Se identificaron 51 especies en Ventanas y nueve en Carbón (Cuadro 1), donde predominaron los gasterópodos de las familias Columbellidae y Muricidae. En el infralitoral destacó los bivalvos Chama buddiana C. B. Adams, 1852, Barbatia gradata Broderip \& Sowerby, 1829 y Arca mutabilis (G. B. Sowerby I, 1833), que no se presentaron en las playas citadas anteriormente. Las zonas altas del litoral y supralitoral presentaron bivalvos del género Brachidontes y caracoles litorínidos. Además, se observó una gran riqueza de especies de micromoluscos. En playa Carbón se identificaron nueve especies, todos ellos gasterópodos del grupo Heterobranchia, además de Brachidontes y caracoles litorínidos.

Poliquetos: Los poliquetos de estas zonas rocosas (ocho especies en Carbón y cuatro en Ventanas) estuvieron representados por las familias Eunicidae, Nereididae y así como Amphinomidae. Las colonias de Sabellariidae en playa Ventanas estaban bien desarrolladas siendo, en apariencia, de las más saludables del Pacífico Norte de Costa Rica.

Otros: En Ventanas se pudo observar una gran abundancia de esponjas y otros invertebrados marinos debajo de las rocas; en Carbón aparecieron pepinos de mar y ascidias.

\section{Sector Cabo Blanco:}

Sector San Miguel, Cabo Blanco: Zona intermareal con plataforma rocosa de arenisca cementada, expuesta a fuerte oleaje en pleamar, de poca pendiente, con una laguna intermareal con cantos rodados y zonas de arena submareales. Cuenta con medidas de protección, siendo parte de la Reserva Absoluta Cabo Blanco, y ubicada a $2 \mathrm{~km}$ del poblado más cercano. Las algas tenían baja cobertura, salvo en las zonas del infralitoral más expuestas al oleaje, donde dominaban las algas verdes frondosas como Ulva. El género Padina también, se encontraba formando parches en dicho estrato. En las zonas más expuestas en la marea baja del infralitoral y litoral bajo aparecieron algas rojas con formas frondosas e incrustantes; entre ellas algas calcáreas (ej. Amphiroa apareció frecuentemente con una cobertura media, 50\%), algunas cubriendo zonas de litoral con muy baja cobertura. Asimismo, las cianobacterias tapizaban gran parte de la roca sumergida.

Crustáceos: Las poblaciones fueron muy diversas y abundantes (40 especies, Cuadro 1). Esta localidad ha sido la que ha presentado un mayor número de especies dentro de la familia Porcellanidae (12 especies). La banda del cirripedio C. panamensis era inexistente en las areniscas, pero algunos cirripedios de esta familia fueron encontrados en grietas.

Moluscos: La riqueza de especies (96 especies, Cuadro 1) y su abundancia fue más alta que en las playas anteriores. Los bivalvos dominantes en el infralitoral fueron C. echinata, el perforador Lithophaga, los bivalvos de importancia comercial P. mazatlanica y $P$. rugosa, y el quitón Chiton stokesii Broderip in Broderip \& Sowerby, 1832, que frecuentemente fue encontrado entre las grietas. En el muestreo realizado en la gran poza de marea formada 
casi al frente de la estación de guardaparques, se pudieron contar al menos seis individuos de la especie Strombus galeatus Swainson, 1823 en estado adulto, especie protegida en el país. Entre las algas verdes se encontraron dos especies de gasterópodos sacoglosos nuevas para la ciencia. Del litoral medio al supralitoral aparecieron varios gastrópodos, con densidad alta y tallas grandes, como las lapas con hueco F. virescens y las lapas pulmonadas Siphonaria gigas G. B. Sowerby I, 1825; estas últimas presentaron un diámetro mayor a $5 \mathrm{~cm}$. La zona supralitoral presentó densidades muy altas de Littorinidae y el nerítido Nerita scabricosta Lamarck, 1822.

Poliquetos: El sector de San Miguel fue, junto a Cabuya, el sitio con una mayor diversidad de este grupo (30 especies), (Cuadro 1). Están presentes las familias Eunicidae, Nereididae, Terebellidae, Capitellidae, Amphinomidae, Sabellidae, Cirratulidae, Opheliidae, Spionidae, Syllidae, Polynoidae, Flabelligeridae y Hesionidae. Las colonias de sabeláridos no fueron muy abundantes en función de su cobertura, si bien se encontraron en buen estado en la punta rocosa más al sur de San Miguel.

Otros: Se recolectaron equinodermos y ascidias, además de cinco taxones de nemertinos y los sipuncúlidos A. antillarum y Phascolosoma perlucens Baird, 1868.

Cabuya, Cabo Blanco: La zona está protegida por ser parte de la Reserva Absoluta Cabo Blanco, y está ubicada a 700m del poblado más cercano. Es un intermareal rocoso, de areniscas, con muchos bloques y cantos rodados y algunos basaltos en la parte supralittoral. Pese a poseer medidas de conservación, la riqueza de especies de invertebrados resultó baja al estar sometido a alto grado de sedimentación de modo natural, producto de los derrumbes de la pared tras la playa por el golpe del oleaje en marea alta (ej. importantes desprendimientos ocurrieron en la zona después de un fuerte temporal en el 2011). El infralitoral y litoral bajo presentó algas como Padina y Halimeda. Otras algas verdes y rojas frondosas presentaron una cobertura media (50\%) mientras que las incrustantes fueron poco frecuentes $(\leq 20 \%)$.

Crustáceos: Se presentaron solo nueve familias de crústaceos y 20 especies (Cuadro 1). Sí se observó una alta densidad de estomatópodos (i.e. Neogonodactylus festae (Nobili, 1901) y Neogonodactylus zacae (Manning, 1972)), de talla grande y que se encontraban cazando activamente en el momento del muestreo. Entre los cirripedios sólo se encontraron E. rhizophorae y C. panamensis.

Moluscos: Se identificaron un total de 15 especies (Cuadro 1). En el litoral bajo aparecieron perforaciones del bivalvo Lithophaga. Además, al igual que en las otras localidades, aparecieron representantes de caracoles Littorinidae y Neritidae, pero en muy baja densidad. No se detectó la presencia de lapas pulmonadas.

Poliquetos: Se encontraron, al igual que en San Miguel, un total de 30 especies (Cuadro 1), correspondiendo, entre otras familias, a Terebellidae, Trichobranchidae, Eunicidae, Nereididae, Syllidae, Dorvilleidae y Chrysopetalidae. Las colonias de Sabellariidae se encontraban en este caso en condiciones óptimas en el litoral.

Otros: Solamente se encontraron dos taxones diferentes de nemertinos y tres sipuncúlidos de la familia Phascolosomatidae.

Tambor: En la playa hay una zona intermareal rocosa, de bloques y cantos rodados de basalto. La zona presenta poca influencia del oleaje en el interior occidental de la Bahía. Es una zona no protegida, a 300 metros del poblado más cercano, y en la cercanía existe un restaurante y muelle sobre pilotes, quizá responsables de que se encontrara basura a lo largo de la zona rocosa.

Crustáceos: A pesar de ser una playa contaminada, y en la cual el tiempo para tomar las muestras fue corto, se recolectó una importante cantidad de familias y especies comparado con otros sitios y áreas protegidas, en particular de la familia Porcellanidae, con cuatro géneros, nueve especies y una alta abundancia. Para el resto de familias, la densidad de las poblaciones fue baja. Además, se encontraron cuatro especies de cirripedios:: en el litoral bajo $B$. 
inexpectatus y $T$. stalactifera en la zona del muelle, en el litoral medio C. panamensis, y en el litoral alto E. rhizophorae.

Moluscos: El bivalvo P. mazatlanica fue comunmente encontrado entre las rocas. Se identificaron en total 15 especies de gasterópodos, entre ellas tres que no habían sido encontradas en las localidades visitadas en 2012, incluyendo moluscos heterobranquios. En dicha zona rocosa las lapas pulmonadas, $S$. gigas, eran de tallas muy pequeñas y escasas.

Poliquetos: No se encontraron individuos en esta localidad, ni colonias de sabeláridos.

Otros: Los sipuncúlidos del género Phascolosoma aparecieron en las grietas de las rocas pero en menor densidad que en otras zonas rocosas estudiadas. La presencia de equinodermos (estrellas quebradizas y pepinos de mar) fue asimismo muy baja (Cuadro 1).

Estudios previos en costa rocosa: Entre los sitios con datos tomados antes de 2012, presentados en este estudio, destacan Sámara y Virador. En la primera se registraron 65 especies de moluscos marinos y 15 de crustáceos (Cuadro 1) siendo la segunda playa no protegida con una mayor riqueza de este grupo después de Playa Matapalo. En Playa Virador la riqueza fue de fue diez especies de moluscos y sólo cuatro de crustáceos, pero hay que tener en cuenta que en este caso, sólo se estudió la epifauna (Cuadro 1).

En el Cuadro 2 se presentan los estudios realizados en las zonas intermareales rocosas del Pacífico Norte de Costa Rica. En dichos

\section{CUADRO 2}

Riqueza de especies de crustáceos, moluscos, poliquetos y otros invertebrados en las costas rocosas del Pacífico Norte, reportadas en trabajos anteriores. Las coordenadas son aproximadas, basadas en los mapas o descripciones de los sitios de muestreo en las referencias. ZP: Zona protegida por el Ministerio de Ambiente, NP: Zona no protegida. Las localidades están ordenadas de Norte a Sur

\begin{tabular}{|c|c|c|c|c|c|c|c|}
\hline Sitio & $\begin{array}{l}\text { Grupo } \\
\text { taxonómico }\end{array}$ & Clases & Familias & Géneros & Especies & $\begin{array}{c}\text { Grupo } \\
\text { estudiado }\end{array}$ & Referencia \\
\hline \multirow{4}{*}{$\begin{array}{l}\text { La Coyotera a, Bahía Salinas } \\
11^{\circ} 1.630^{\prime} \mathrm{N}-85^{\circ} 42.724^{\prime} \mathrm{W}-\mathrm{NP}\end{array}$} & Crustacea & 1 & 2 & 3 & 3 & \multirow[t]{4}{*}{ epifauna } & \multirow{4}{*}{$\begin{array}{l}\text { Sibaja-Cordero \& Cortés } \\
\text { (2008) }\end{array}$} \\
\hline & Mollusca & 2 & 5 & 5 & 5 & & \\
\hline & Polychaeta & 0 & 0 & 0 & 0 & & \\
\hline & Otros & 1 & 1 & 1 & 1 & & \\
\hline \multirow{4}{*}{$\begin{array}{l}\text { La Coyotera b, Bahía Salinas } \\
11^{\circ} 1.999^{\prime} \mathrm{N}-85^{\circ} 43.075^{\prime} \mathrm{W}-\mathrm{NP}\end{array}$} & Crustacea & 2 & 4 & 5 & 5 & \multirow[t]{4}{*}{ epifauna } & \multirow{4}{*}{$\begin{array}{l}\text { Sibaja-Cordero \& Cortés } \\
\text { (2008) }\end{array}$} \\
\hline & Mollusca & 3 & 6 & 6 & 6 & & \\
\hline & Polychaeta & 0 & 0 & 0 & 0 & & \\
\hline & Otros & 1 & 1 & 1 & 1 & & \\
\hline \multirow{4}{*}{$\begin{array}{l}\text { Jobo a, Bahía Salinas } \\
11^{\circ} 2.785^{\prime} \mathrm{N}-85^{\circ} 43.883^{\prime} \mathrm{W}-\mathrm{NP}\end{array}$} & Crustacea & 1 & 2 & 3 & 3 & \multirow[t]{4}{*}{ epifauna } & \multirow{4}{*}{$\begin{array}{l}\text { Sibaja-Cordero \& Cortés } \\
\text { (2008) }\end{array}$} \\
\hline & Mollusca & 3 & 9 & 9 & 9 & & \\
\hline & Polychaeta & 0 & 0 & 0 & 0 & & \\
\hline & Otros & 1 & 1 & 1 & 1 & & \\
\hline \multirow{4}{*}{$\begin{array}{l}\text { Jobo b, Bahía Salinas } \\
11^{\circ} 2.806^{\prime} \mathrm{N}-85^{\circ} 44.015^{\prime} \mathrm{W}-\mathrm{NP}\end{array}$} & Crustacea & 2 & 3 & 4 & 4 & \multirow[t]{4}{*}{ epifauna } & \multirow{4}{*}{$\begin{array}{l}\text { Sibaja-Cordero \& Cortés } \\
\text { (2008) }\end{array}$} \\
\hline & Mollusca & 3 & 9 & 10 & 10 & & \\
\hline & Polychaeta & 0 & 0 & 0 & 0 & & \\
\hline & Otros & 1 & 1 & 1 & 1 & & \\
\hline $\begin{array}{l}\text { Junquillal } \\
10^{\circ} 57.803^{\prime} \mathrm{N}-85^{\circ} 41.571^{\prime} \mathrm{W}-\mathrm{ZP}\end{array}$ & Crustacea & 1 & 1 & 1 & 1 & $\begin{array}{l}\text { Fauna } \\
\text { endolítica }\end{array}$ & Fischer (1981) \\
\hline $\begin{array}{l}\text { Parker, Santa Elena } \\
10^{\circ} 55.288^{\prime} \mathrm{N}-85^{\circ} 48.687^{\prime} \mathrm{W}-\mathrm{ZP}\end{array}$ & Polychaeta & 1 & 2 & 4 & 4 & Poliquetos & Dean (2004) \\
\hline
\end{tabular}


CUADRO 2 (Continuación)

\begin{tabular}{|c|c|c|c|c|c|c|c|}
\hline Sitio & $\begin{array}{c}\text { Grupo } \\
\text { taxonómico }\end{array}$ & Clases & Familias & Géneros & Especies & $\begin{array}{c}\text { Grupo } \\
\text { estudiado }\end{array}$ & Referencia \\
\hline $\begin{array}{l}\text { Playa Blanca, Santa Elena } \\
10^{\circ} 55.810^{\prime} \mathrm{N}-85^{\circ} 51.808^{\prime} \mathrm{W}-\mathrm{ZP}\end{array}$ & Polychaeta & 1 & 1 & 1 & 1 & Poliquetos & Dean (2004) \\
\hline $\begin{array}{l}\text { Islas Murciélagos } \\
10^{\circ} 51.376^{\prime} \mathrm{N}-85^{\circ} 54.862^{`} \mathrm{~W}-\mathrm{ZP}\end{array}$ & Otros & 1 & 1 & 2 & 2 & Sipuncula & Dean (2001a) \\
\hline Bahía Culebra - NP & Polychaeta & 1 & 1 & 1 & 1 & Poliquetos & Dean (2004) \\
\hline \multirow{2}{*}{$\begin{array}{l}\text { Panamá } \\
10^{\circ} 35.700^{`} \mathrm{~N}-85^{\circ} 41.000^{\prime} \mathrm{W}-\mathrm{NP}\end{array}$} & Polychaeta & 1 & 1 & 2 & 2 & Poliquetos & Dean (2001b) \\
\hline & Otros & 1 & 1 & 1 & 1 & Sipuncula & $\operatorname{Dean}(2001 \mathrm{a})$ \\
\hline $\begin{array}{l}\text { Hermosa, norte } \\
10^{\circ} 34.864^{\prime} \mathrm{N}-85^{\circ} 40.594^{\prime} \mathrm{W}-\mathrm{NP}\end{array}$ & Mollusca & 2 & 11 & 11 & 15 & Moluscos & Miller (1974) \\
\hline $\begin{array}{l}\text { Hermosa, sur } \\
10^{\circ} 34.227^{\prime} \mathrm{N}-85^{\circ} 41.083^{\prime} \mathrm{W}-\mathrm{NP}\end{array}$ & Mollusca & 2 & 18 & 24 & 35 & Moluscos & Miller (1974) \\
\hline Playa del Coco - NP & Polychaeta & 1 & 1 & 2 & 3 & Poliquetos & Dean (2004) \\
\hline \multirow{4}{*}{$\begin{array}{l}\text { Playa del Coco } \\
10^{\circ} 33.216^{\prime} \mathrm{N}-85^{\circ} 42.491^{\prime} \mathrm{W}-\mathrm{NP}\end{array}$} & Crustacea & 0 & 0 & 0 & 0 & \multirow{4}{*}{$\begin{array}{l}\text { Fauna } \\
\text { endolítica en } \\
\text { basalto }\end{array}$} & \multirow{4}{*}{ Pepe (1985) } \\
\hline & Mollusca & 1 & 1 & 1 & 1 & & \\
\hline & Polychaeta & 1 & 1 & 2 & 2 & & \\
\hline & Otros & 1 & 2 & 2 & 3 & & \\
\hline \multirow{4}{*}{$\begin{array}{l}\text { Playa del Coco } \\
10^{\circ} 33.312^{\prime} \mathrm{N}-85^{\circ} 42.511^{\prime} \mathrm{W}-\mathrm{NP}\end{array}$} & Crustacea & 0 & 0 & 0 & 0 & \multirow{4}{*}{$\begin{array}{l}\text { Fauna } \\
\text { endolítica en } \\
\text { coral muerto }\end{array}$} & \multirow{4}{*}{ Pepe (1985) } \\
\hline & Mollusca & 1 & 1 & 1 & 1 & & \\
\hline & Polychaeta & 1 & 1 & 2 & 2 & & \\
\hline & Otros & 2 & 3 & 3 & 4 & & \\
\hline \multirow{3}{*}{$\begin{array}{l}\text { Playa del Coco } \\
10^{\circ} 33.389^{\prime} \mathrm{N}-85^{\circ} 42.735^{\prime} \mathrm{W} \text { a } \\
10^{\circ} 33.218^{\prime} \mathrm{N}-85^{\circ} 42.492^{\prime} \mathrm{W}-\mathrm{NP}\end{array}$} & Crustacea & 1 & 4 & 5 & 5 & \multirow[t]{3}{*}{ Moluscos } & \multirow{3}{*}{$\begin{array}{l}\text { Spight }(1976 ; 1977 \text {; } \\
\text { 1978) }\end{array}$} \\
\hline & Mollusca & 2 & 28 & 51 & 80 & & \\
\hline & Otros & 4 & 4 & 4 & 6 & & \\
\hline $\begin{array}{l}\text { Punta Cirial, Ocotal } \\
10^{\circ} 32.880^{\prime} \mathrm{N}-85^{\circ} 43.566^{\prime} \mathrm{W}-\mathrm{NP}\end{array}$ & Crustacea & 1 & 1 & 1 & 1 & $\begin{array}{l}\text { Fauna } \\
\text { endolítica }\end{array}$ & Fischer (1981) \\
\hline Conchal - NP & Otros & 1 & 1 & 2 & 2 & Sipuncula & Dean (2001a) \\
\hline \multirow{3}{*}{$\begin{array}{l}\text { Punta San Francisco, Tamarindo } \\
10^{\circ} 17.723^{\prime} \mathrm{N}-85^{\circ} 51.147^{\prime} \mathrm{W}-\mathrm{NP}\end{array}$} & Crustacea & 1 & 1 & 1 & 1 & \multirow{3}{*}{$\begin{array}{l}\text { Fauna } \\
\text { endolítica }\end{array}$} & \multirow[t]{3}{*}{ Fischer (1981) } \\
\hline & Mollusca & 1 & 1 & 1 & 1 & & \\
\hline & Otros & 1 & 1 & 1 & 1 & & \\
\hline $\begin{array}{l}\text { Nosara } \\
9^{\circ} 57.328^{\prime} \mathrm{N}-85^{\circ} 40.540^{\prime} \mathrm{W}-\mathrm{NP}\end{array}$ & Otros & 1 & 1 & 1 & 1 & $\begin{array}{l}\text { Fauna } \\
\text { endolítica }\end{array}$ & Fischer (1981) \\
\hline Sámara - NP & Mollusca & 1 & 1 & 1 & 1 & Sipuncula & Dean (2001a) \\
\hline $\begin{array}{l}\text { Cangrejal, Sámara } \\
9^{\circ} 52.497^{\prime} \mathrm{N}-85^{\circ} 32.010^{\prime} \mathrm{W}-\mathrm{NP}\end{array}$ & Mollusca & 1 & 7 & 8 & 9 & Quitones & Jörger et al. (2008) \\
\hline $\begin{array}{l}\text { Sámara } \\
9^{\circ} 52.433{ }^{\prime} \mathrm{N}-85^{\circ} 32.133^{\prime} \mathrm{W}-\mathrm{NP}\end{array}$ & Mollusca & 2 & 34 & 28 & 53 & Moluscos & Miller (1974) \\
\hline $\begin{array}{l}\text { Punta Indio, Carrillo } \\
9^{\circ} 51.677^{\prime} \mathrm{N} 85^{\circ} 29.895^{\prime} \mathrm{W}-\mathrm{NP}\end{array}$ & Mollusca & 1 & 1 & 1 & 1 & $\begin{array}{l}\text { Fauna } \\
\text { endolitica }\end{array}$ & Fischer (1981) \\
\hline Cabo Blanco - ZP & Otros & 1 & 1 & 1 & 1 & Sipuncula & Dean (2001a) \\
\hline \multirow{2}{*}{$\begin{array}{l}\text { Montezuma } \\
9^{\circ} 39.615^{\prime} \mathrm{N}-85^{\circ} 3.525^{\prime} \mathrm{W}-\mathrm{NP}\end{array}$} & Crustacea & 1 & 2 & 2 & 2 & \multirow[t]{2}{*}{ epifauna } & \multirow[t]{2}{*}{ Villalobos (1980a) } \\
\hline & Mollusca & 2 & 3 & 4 & 4 & & \\
\hline
\end{tabular}


CUADRO 2 (Continuación)

\begin{tabular}{|c|c|c|c|c|c|c|c|}
\hline Sitio & $\begin{array}{c}\text { Grupo } \\
\text { taxonómico }\end{array}$ & Clases & Familias & Géneros & Especies & $\begin{array}{c}\text { Grupo } \\
\text { estudiado }\end{array}$ & Referencia \\
\hline \multirow{4}{*}{$\begin{array}{l}\text { Montezuma } \\
9^{\circ} 39.146^{\prime} \mathrm{N}-85^{\circ} 4.051^{\prime} \mathrm{W}-\mathrm{NP}\end{array}$} & Crustacea & 1 & 2 & 2 & 2 & \multirow[t]{4}{*}{ epifauna } & \multirow{4}{*}{$\begin{array}{l}\text { Sibaja-Cordero \& } \\
\text { Vargas Zamora (2006) }\end{array}$} \\
\hline & Mollusca & 2 & 5 & 5 & 6 & & \\
\hline & Polychaeta & 0 & 0 & 0 & 0 & & \\
\hline & Otros & 1 & 1 & 1 & 1 & & \\
\hline \multirow{4}{*}{$\begin{array}{l}\text { Tambor, muelle } \\
9^{\circ} 42.818^{\prime} \mathrm{N}-85^{\circ} 0.637^{\prime} \mathrm{W}-\mathrm{NP}\end{array}$} & Crustacea & 1 & 2 & 3 & 4 & \multirow[t]{4}{*}{ epifauna } & \multirow[t]{4}{*}{ Villalobos (1980b) } \\
\hline & Mollusca & 1 & 6 & 7 & 7 & & \\
\hline & Polychaeta & 1 & 1 & 1 & 1 & & \\
\hline & Otros & 0 & 0 & 0 & 0 & & \\
\hline \multirow{4}{*}{$\begin{array}{l}\text { Tambor } \\
9^{\circ} 42.799^{\prime} \mathrm{N}-85^{\circ} 0.845^{\prime} \mathrm{W}-\mathrm{NP}\end{array}$} & Crustacea & 1 & 2 & 3 & 3 & \multirow[t]{4}{*}{ epifauna } & \multirow[t]{4}{*}{ Villalobos (1980b) } \\
\hline & Mollusca & 2 & 9 & 10 & 10 & & \\
\hline & Polychaeta & 1 & 1 & 1 & 1 & & \\
\hline & Otros & 0 & 0 & 0 & 0 & & \\
\hline
\end{tabular}

estudios, las playas del Coco y Sámara son las que reportaron más especies de moluscos $(>50)$; mientras que el resto presentaron menos de 15 especies. Para los crustáceos, en todos estos estudios se detectaron de cinco a menos especies, y en su mayoría sólo fueron indicados los cirripedios. En el caso de poliquetos y otros invertebrados, constata que los datos son escasos (seis especies como máximo, Cuadro 2). Esto,,indica la poca importancia que se le ha dado a los grupos de invertebrados móviles en dicho ambiente, si se compara con los datos obtenidos en los muestreos de 2012 (Cuadro 1).

La Figura 1 muestra la totalidad de especies por localidad de los estudios que mencionan la zona intermareal rocosa, incluyéndose la información recolectada; en el presente estudio. No existe un patrón espacial en la distribución de la riqueza, distinguiéndose localidades con elevada riqueza de especies en cada una de las secciones del Pacífico Norte. San Miguel de Cabo Blanco es el sitio más diverso con 175 especies, seguido por Matapalo con 130 y Sámara y Ventanas (con 80 especies).

\section{Playas de arena}

\section{Sector Santa Elena:}

Playa Junquillal: Playa de arena de pendiente moderada (intermedia), arena blanca y fina. Es una zona protegida dentro del Sector Junquillal de la ACG, a $2 \mathrm{~km}$ del poblado más cercano. Se encontró sólo cerca del infralitoral y del litoral bajo, cangrejos anomuros (género Hippa), el anfioxo Branchiostoma californiense Andrews, 1893, gastrópodos de la familia Olividae, y poliquetos pertenecientes a las familias Magelonidae (género Magelona) y a la familia Glyceridae.

\section{Sector Golfo de Papagayo:}

Playa Hermosa: Playa disipativa ubicada frente al poblado, de arena gris y fina, resguardada del oleaje fuerte y que no presenta medidas de conservación. Es mucho menos diversa que otras playas del país y cuenta con cumáceos y nemertinos en su infralitoral; en el litoral, se encontraron poliquetos de las familias Glyceridae y Spionidae (entre estos últimos la especie Scolelepis squamata (O.F. Muller, 1806)). Finalmente, en el litoral alto y supralitoral se encontraron isópodos de la familia Cirolanidae y larvas de dípteros de la familia Dolichopodidae.

Playa Matapalo. La playa es reflectiva, expuesta al oleaje, de arena clara de grano fino a grueso. Es contigua a un desarrollo turístico de gran escala y no cuenta con medidas de conservación. Se encontró una abundancia 


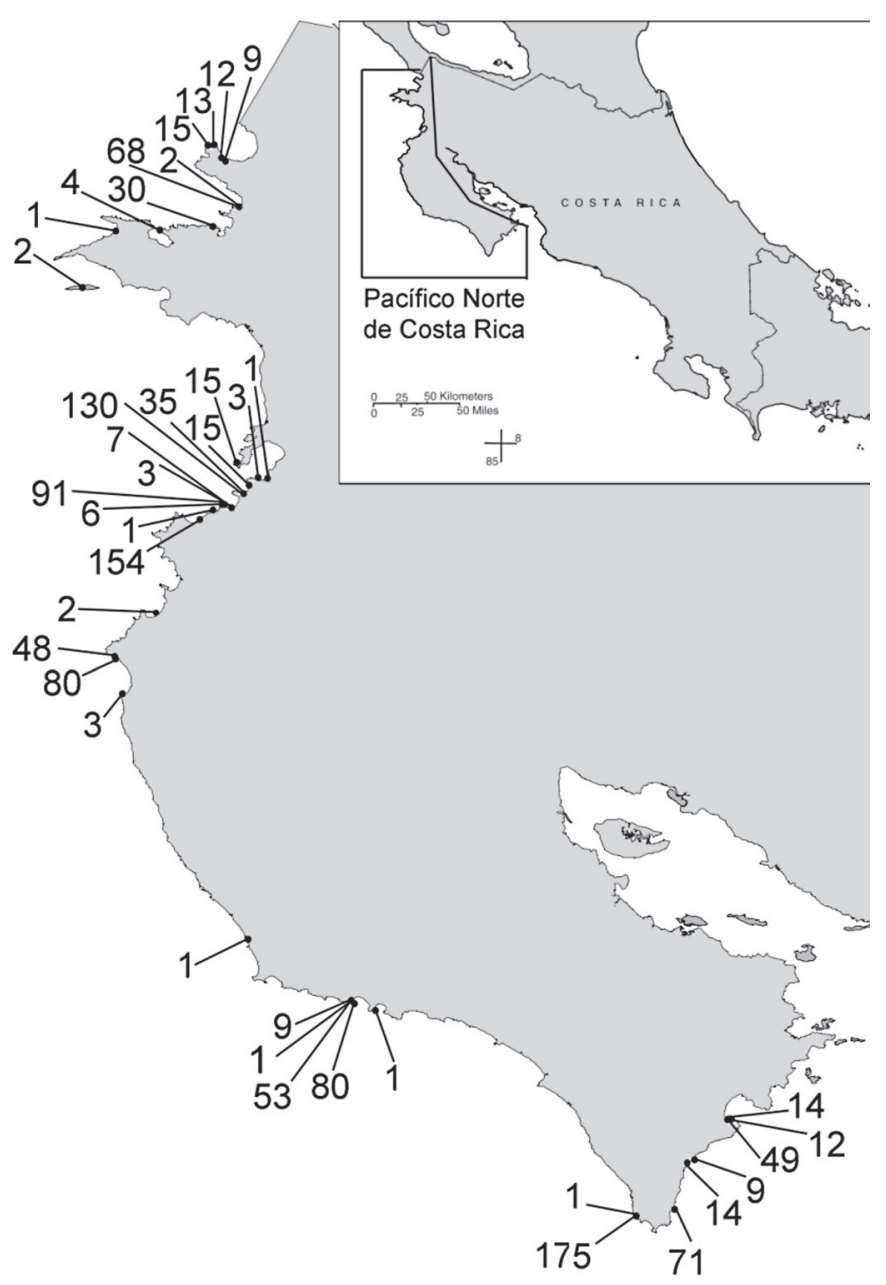

Fig. 1. Riqueza de especies de invertebrados en el intermareal de las costas rocosas del Pacífico Norte de Costa Rica. Según el número de especies, las localidades en el mapa de norte a sur, Sector Santa Elena = 9: La Coyotera a; 12: La Coyotera b; 13: Jobo a; 15: Jobo b; 2: Junquillal; 68: Junquillal; 29: El Hachal; 4: Parker bay; 1: Playa Blanca; 2: Islas Murciélagos; Sector Golfo de Papagayo = 1: Bahía Culebra; 3: Panamá; 15: Virador; 15: Hermosa, norte; 35: Hermosa, sur; 130: Hermosa; 7: Playa del Coco, coral muerto; 3: Playa del Coco; 91: Playa del Coco, Punta Miga a Punta Centinela; 6: Playa del Coco, basalto; 1: Punta Cirial, Ocotal; 154: Matapalo; Sector Punta Gorda-Punta Pargos = 2: Conchal; 48: Carbón; 80: Ventanas; 3: Punta San Francisco, Tamarindo; 1: Nosara; 9: Cangrejal, Sámara; 1: Cangrejal, Sámara; 53: Cangrejal, Sámara; 80: Cangrejal, Sámara; 1: Punta Indio, Carrillo; Sector Cabo Blanco = 1: Cabo Blanco; 172: Cabo Blanco (Sector San Miguel); 71: Cabo Blanco (Sector Cabuya); 14: Montezuma; 9: Montezuma; 49: Tambor; 12: Tambor, muelle; 14: Tambor.

muy baja de organismos, en el infralitoral el poliqueto Magelona y el anomuro Hippa, en el litoral bajo y alto los poliquetos de las familias Lumbrineridae, Glyceridae y Spionidae $(S$. squamata), y en el supralitoral principalmente isópodos cirolánidos.

\section{Sector Punta Gorda-Punta Pargos:}

Playa Ventanas. Playa disipativa de arena blanca y fina, situada dentro del Parque Nacional Marino Las Baulas, a $250 \mathrm{~m}$ del poblado más cercano. El estrato infralitoral 
presentó diversos anfípodos, poliquetos del género Magelona y de las familias Orbiniidae, Spionidae y Paraonidae. En el litoral bajo aparecieron las familias Glyceridae y Paraonidae, además de anfípodos, y los isópodos de la familia Cirolanidae aparecieron a lo largo de todo el intermareal.

\section{Sector Cabo Blanco:}

Playa Tambor. Playa disipativa con arena gris de grano fino. La localidad no es una zona protegida, está a $200 \mathrm{~m}$ del poblado más cercano. En el infralitoral se encontraron, en baja abundancia, nematodos y poliquetos del género Magelona. En el litoral bajo estaban los poliquetos de la familia Spionidae como
Malacoceros sp. En el litoral medio había una franja a media marea poblada por poliquetos tubícolas de la familia Onuphidae, y además, se halló un individuo del pez del género Gobius. En el litoral alto aparecieron bivalvos del género Tellina y de la familia Semelidae, poliquetos de las familias Glyceridae, Pilargidae, Spionidae (S. squamata) y el Opheliidae (Armandia brevis (Moore, 1906) apareció de forma común). Aparecieron isópodos de la familia Cirolanidae y en el supralitoral se encontraron los cangrejos del género Uca.

Estudios previos en playas de arena: $\mathrm{El}$ Cuadro 3 y la Figura 2, presentan el número de especies por playa de arena, encontrados tanto en el presente trabajo (2012) como en trabajos

\section{CUADRO 3}

Riqueza de especies de crustáceos, moluscos, poliquetos y otros invertebrados en las playas de arena del Pacífico Norte, recolectadas en el presente estudio y en trabajos anteriores. Las coordenadas en los estudios previos son aproximadas, frente o al centro de la playa estudiada. ZP: Zona protegida por el Ministerio de Ambiente, NP: Zona no protegida.

Las localidades están ordenadas de Norte a Sur

\begin{tabular}{|c|c|c|c|c|c|c|c|}
\hline Sitio & $\begin{array}{l}\text { Densidad } \\
\text { (ind } / \mathrm{m}^{2} \text { ) }\end{array}$ & $\begin{array}{l}\text { Grupo } \\
\text { taxonómico }\end{array}$ & Clases & Familias & Géneros & Especies & Referencia \\
\hline Junquillal & $178(0-556)$ & Crustacea & 1 & 1 & 1 & 1 & Presente trabajo \\
\hline \multirow[t]{3}{*}{$10^{\circ} 57.969^{\prime} \mathrm{N}-85^{\circ} 41.354^{\prime} \mathrm{W}-\mathrm{ZP}$} & & Mollusca & 1 & 1 & 1 & 1 & \\
\hline & & Polychaeta & 1 & 2 & 2 & 2 & \\
\hline & & Otros & 1 & 1 & 1 & 1 & \\
\hline & \multicolumn{3}{|c|}{$10^{\circ} 55.810^{\prime} \mathrm{N}-85^{\circ} 51.808^{\prime} \mathrm{W}-\mathrm{ZP}$} & 2 & 2 & 2 & Dean (2004) \\
\hline Hermosa & $356(0-889)$ & Crustacea & 1 & 2 & 2 & 2 & Presente trabajo \\
\hline \multirow[t]{3}{*}{$10^{\circ} 34.300^{\prime} \mathrm{N}-85^{\circ} 40.911^{\prime} \mathrm{W}-\mathrm{NP}$} & & Mollusca & 0 & 0 & 0 & 0 & \\
\hline & & Polychaeta & 1 & 2 & 2 & 2 & \\
\hline & & Otros & 1 & 2 & 2 & 2 & \\
\hline Playa del Coco & 1960 & Crustacea & 1 & 7 & 7 & 7 & Dexter (1974) \\
\hline \multirow[t]{3}{*}{$10^{\circ} 33.085^{\prime} \mathrm{N}-85^{\circ} 41.984^{\prime} \mathrm{W}-\mathrm{NP}$} & & Mollusca & 0 & 0 & 0 & 0 & \\
\hline & & Polychaeta & 1 & 4 & 4 & 4 & \\
\hline & & Otros & 1 & 2 & 2 & 2 & \\
\hline \multicolumn{8}{|l|}{$10^{\circ} 31.797^{\prime} \mathrm{N}-85^{\circ} 45.123^{\prime} \mathrm{W}-\mathrm{NP}$} \\
\hline Matapalo & $222(111-333)$ & Crustacea & 1 & 2 & 2 & 2 & Presente trabajo \\
\hline \multirow{3}{*}{$10^{\circ} 31.958^{\prime} \mathrm{N}-85^{\circ} 44.799^{\prime} \mathrm{W}-\mathrm{NP}$} & & Mollusca & 0 & 0 & 0 & 0 & \\
\hline & & Polychaeta & 1 & 4 & 4 & 4 & \\
\hline & & Otros & 0 & 0 & 0 & 0 & \\
\hline \multicolumn{8}{|l|}{$\begin{array}{l}\text { Conchal } \\
10^{\circ} 24.061^{\prime} \mathrm{N}-85^{\circ} 48.401^{\prime} \mathrm{W}-\mathrm{NP}\end{array}$} \\
\hline \multicolumn{8}{|c|}{$10^{\circ} 26.142^{\prime} \mathrm{N}-85^{\circ} 47.572^{\prime} \mathrm{W}-\mathrm{NP}$} \\
\hline Ventanas & $4267(0-16444)$ & Crustacea & 1 & 2 & 2 & 2 & Presente trabajo \\
\hline \multirow[t]{3}{*}{$10^{\circ} 20.741^{\prime} \mathrm{N}-85^{\circ} 51.560^{\prime} \mathrm{W}-\mathrm{ZP}$} & & Mollusca & 0 & 0 & 0 & 0 & \\
\hline & & Polychaeta & 1 & 5 & 5 & 5 & \\
\hline & & Otros & 0 & 0 & 0 & 0 & \\
\hline
\end{tabular}


CUADRO 3 (Continuación)

\begin{tabular}{|c|c|c|c|c|c|c|c|}
\hline Sitio & $\begin{array}{l}\text { Densidad } \\
\text { (ind } / \mathrm{m}^{2} \text { ) }\end{array}$ & $\begin{array}{l}\text { Grupo } \\
\text { taxonómico }\end{array}$ & Clases & Familias & Géneros & Especies & Referencia \\
\hline Tamarindo & 847 & Crustacea & 1 & 7 & 7 & 7 & Dexter (1974), \\
\hline \multirow[t]{3}{*}{$10^{\circ} 18.197^{\prime} \mathrm{N}-85^{\circ} 50.414^{\prime} \mathrm{W}-\mathrm{NP}$} & & Mollusca & 2 & 4 & 4 & 5 & Fauchald (1973), \\
\hline & & Polychaeta & 1 & 4 & 4 & 4 & Dean (2001b) \\
\hline & & Otros & 1 & 1 & 1 & 1 & \\
\hline $\begin{array}{l}\text { Guiones } \\
9^{\circ} 56.358^{\prime} \mathrm{N}-85^{\circ} 40.084^{\prime} \mathrm{W}-\mathrm{NP}\end{array}$ & - & Polychaeta & 1 & 1 & 1 & 1 & Böggemann (2002) \\
\hline $\begin{array}{l}\text { Carrillo } \\
9^{\circ} 52.257^{\prime} \mathrm{N}-85^{\circ} 29.623^{\prime} \mathrm{W}-\mathrm{NP}\end{array}$ & - & Polychaeta & 1 & 1 & 1 & 1 & Böggemann (2002) \\
\hline Sámara & 450 & Crustacea & 1 & 4 & 4 & 4 & Dexter (1974), \\
\hline \multirow{3}{*}{$9^{\circ} 52.793^{\prime} \mathrm{N}-85^{\circ} 31.637^{\prime} \mathrm{W}-\mathrm{NP}$} & & Mollusca & 2 & 3 & 4 & 5 & Böggemann (2002) \\
\hline & & Polychaeta & 1 & 3 & 4 & 4 & \\
\hline & & Otros & 1 & 1 & 2 & 2 & \\
\hline $\begin{array}{l}\text { Punta Islita } \\
9^{\circ} 51.141^{\prime} \mathrm{N}-85^{\circ} 24.135^{\prime} \mathrm{W}-\mathrm{NP}\end{array}$ & - & Polychaeta & 1 & 1 & 1 & 1 & Böggemann (2002) \\
\hline $\begin{array}{l}\text { Montezuma } \\
9^{\circ} 39.242^{\prime} \mathrm{N}-85^{\circ} 3.878^{\prime} \mathrm{W}-\mathrm{NP}\end{array}$ & - & Polychaeta & 1 & 1 & 1 & 1 & Böggemann (2002) \\
\hline Tambor & $1622(222-6667)$ & Crustacea & 1 & 3 & 3 & 3 & Presente trabajo \\
\hline \multirow[t]{3}{*}{$9^{\circ} 42.799^{\prime} \mathrm{N}-85^{\circ} 0.920^{\prime} \mathrm{W}-\mathrm{NP}$} & & Mollusca & 1 & 2 & 2 & 2 & \\
\hline & & Polychaeta & 1 & 7 & 8 & 8 & \\
\hline & & Otros & 2 & 2 & 2 & 2 & \\
\hline $\begin{array}{l}\text { Curú } \\
9^{\circ} 47.311^{\prime} \mathrm{N}-84^{\circ} 55.455^{\prime} \mathrm{W}-\mathrm{ZP}\end{array}$ & - & Otros & 1 & 1 & 1 & 1 & Dean (2001b) \\
\hline
\end{tabular}

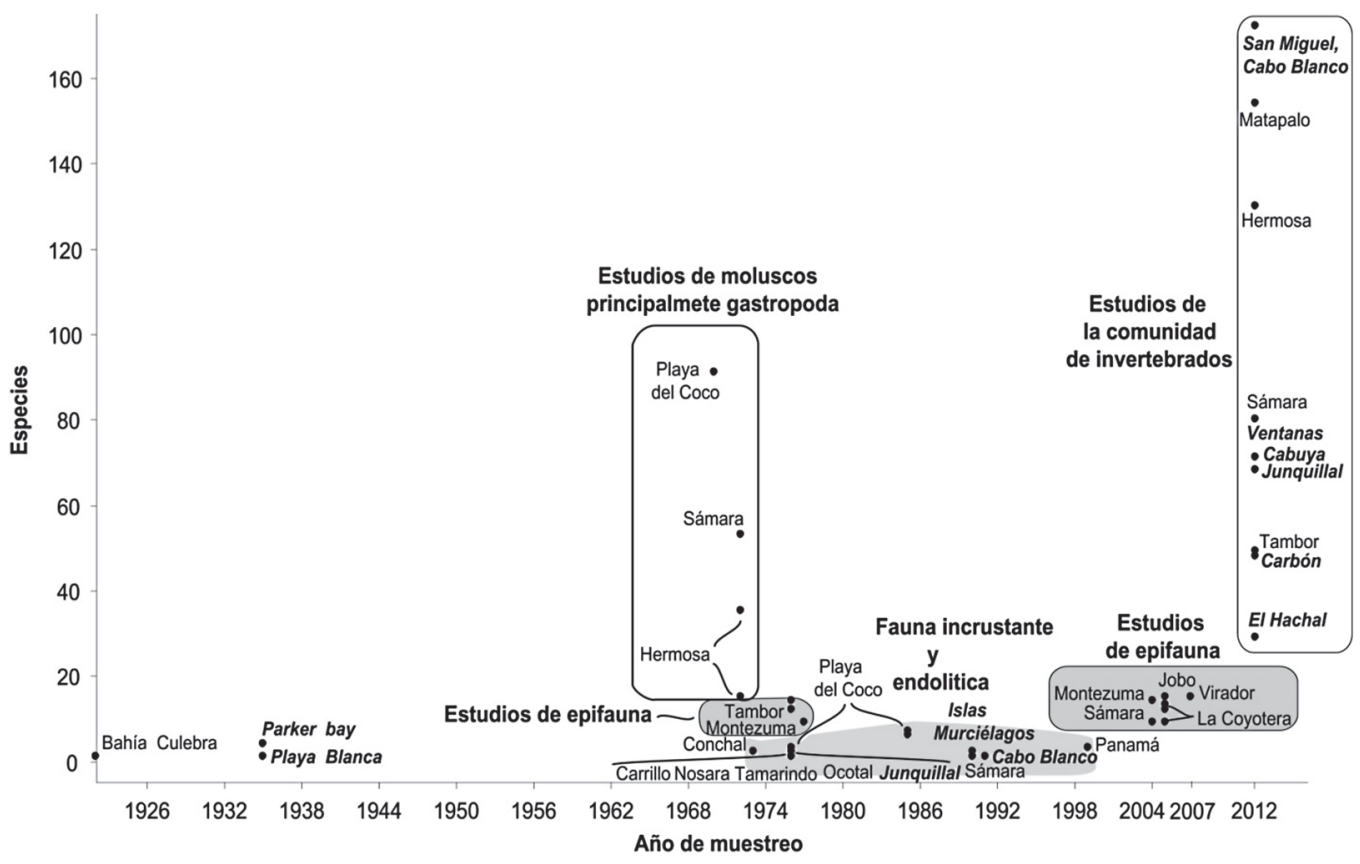

Fig. 2. Número de especies de invertebrados por estudio del intermareal rocoso, según localidad y año de muestreo. Las zonas protegidas se presentan en itálica y negrita. Se agrupan las localidades según el tipo de fauna al que estaba dirigido el estudio (e.g. epifauna). 
previos. De estos Tamarindo, Tambor y Sámara fueron las localidades más ricas en especies, con 17, 15, 15 especies, respectivamente, seguidas por El Coco, Ventanas, Matapalo y Hermosa, con 13, 7, 6 y 6 especies. En el Cuadro 3 se observa que la riqueza de especies por grupo fue baja (Cuadro 3), ocupando el primer lugar los poliquetos mientras que otros son los crustáceos peracáridos los mejor representados, principalmente por anfípodos.

Efecto de las zonas protegidas: El número de especies promedio reportado cuando no hay ninguna medida de conservación en las zonas rocosas intermareales es de 26 , mientras que cuando existe tales medidas, asciende a 43. Debido a la alta variabilidad existente en los datos, la diferencia no resulta significativa $(\mathrm{t}=1.12, \mathrm{p}=0.268$, Fig. $4 \mathrm{~A})$. Se agrupan los trabajos hechos en las zonas rocosas del Pacífico Norte de Costa Rica en varios grupos a través del tiempo (Fig. 3): Los primeros trabajos son recolectas esporádicas, otros estudios solamente cuenta con grupos de la epifauna (con un máximo de 15 especies en las investigaciones. Además, en los estudios donde más especies se

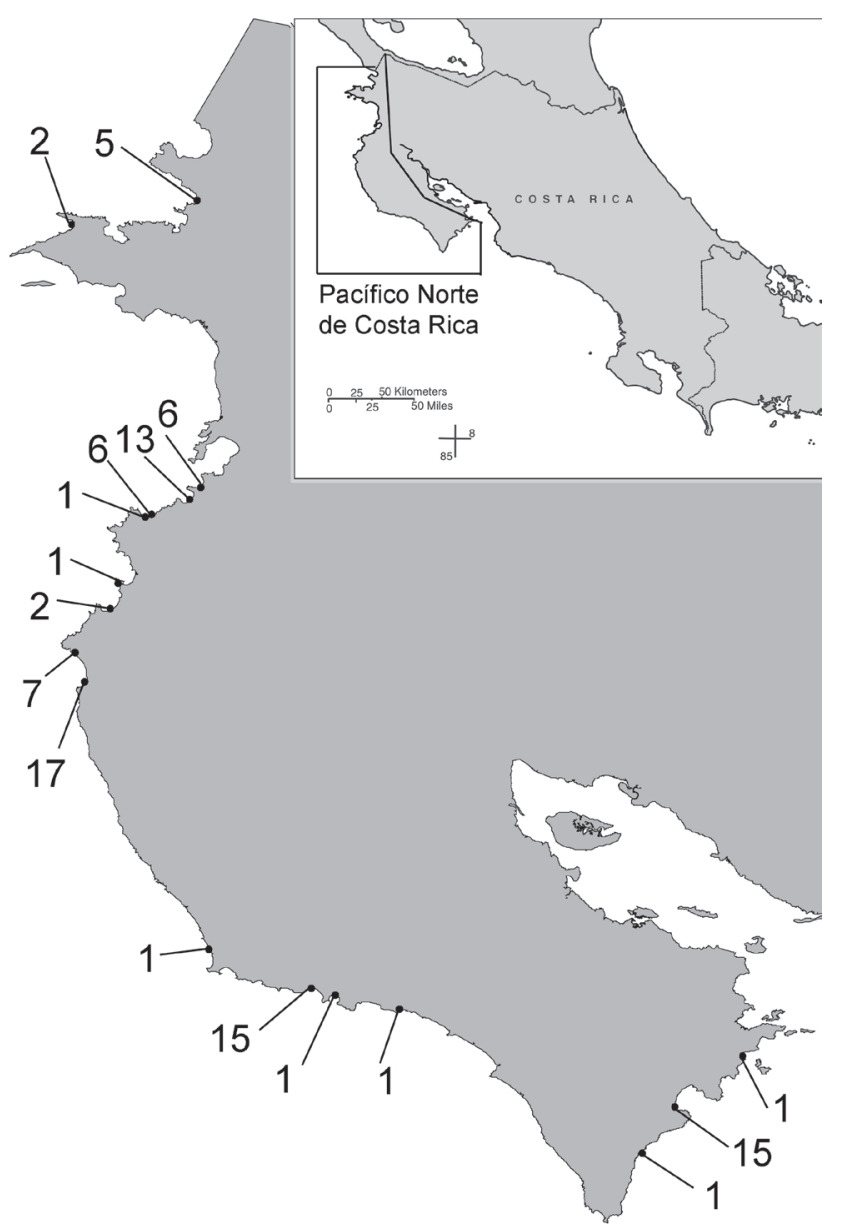

Fig. 3. Riqueza de especies de invertebrados en el intermareal de las playas de arena del Pacífico Norte de Costa Rica. Según el número de especies, las localidades en el mapa de norte a sur y el año del muestreo, Sector Santa Elena = 5: Junquillal, 2012; 2: Playa Blanca, Santa Elena, 1939, 1979; Sector Golfo de Papagayo = 6: Hermosa, 2012; 13, Playa del Coco, 1971; 6: Matapalo, 2012, 1: Matapalo, 1996; Sector Punta Gorda-Punta Pargos = 1: Flamingo, 1996; 2: Conchal, 1976; 7: Ventanas, 2012; 17: Tamarindo, 1971; 1: Guiones, 1996; 1: Carillo, 1996; 15: Sámara, 1971, 1996; 1: Punta Islita, 1997; Sector Cabo Blanco = 1: Montezuma, 1996; 15: Tambor, 2012; 1: Curú, 1984. 

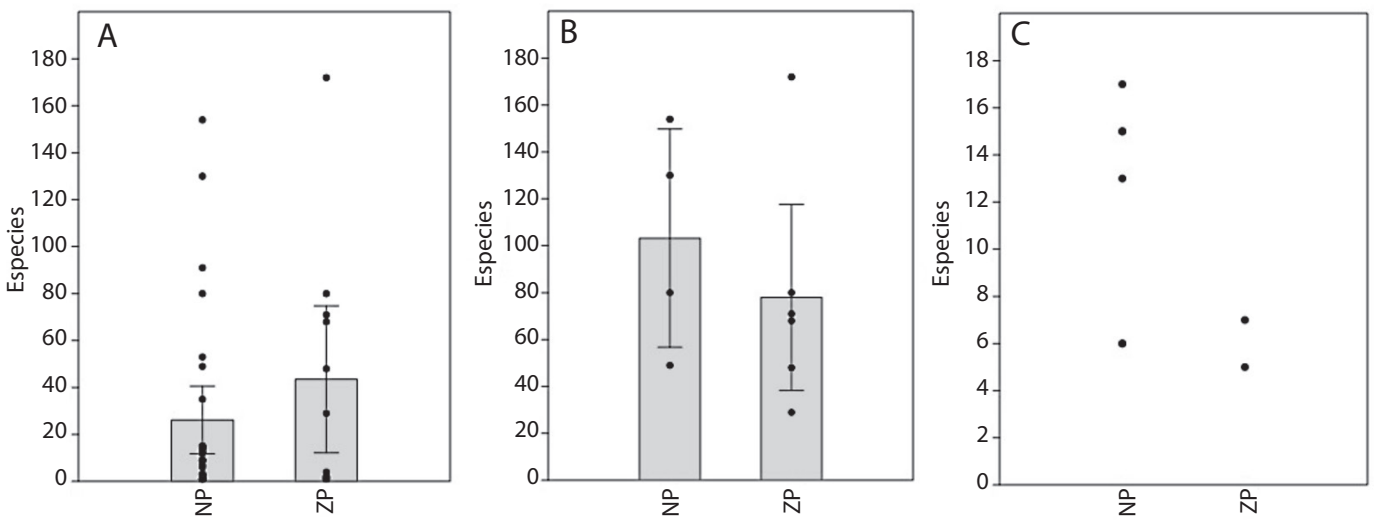

Fig. 4. Riqueza de especies reportadas y su promedio con límites de confianza (95\%), para las zonas sin (NP) y con medidas de protección (ZP), en los intermareales del Pacífico Norte de Costa Rica. A: Todos los estudios en zonas rocosas, incluido el presente B: Datos de las zonas rocosas de entre mareas, visitadas durante 2012 (presente estudio). C: Especies reportadas para las playas de arena por Dexter (1974) y el presente estudio.

reportaron sólo se enfocaron hacia ciertos taxones (e.g. moluscos), y, finalmente el presente trabajo realizado en el 2012 se tomó en cuenta toda la comunidad, presentando más de 29 taxones por localidad (Fig. 3). Cuando solo se consideran los muestreos realizados en el 2012, para calcular el promedio (Fig. 4B), tampoco existen diferencias significativas en el número de taxones entre zonas con medidas de conservación o sin ellas $(\mathrm{t}=0.801, \mathrm{p}<0.446)$. En las playas de arena en la Fig. 4C se observa también, que la mayor diversidad de organismos en los estudios antes mencionados se encuentran reportadas fuera de zonas de conservación. La densidad de individuos fue alta en Playa Ventanas (4267 individuos $/ \mathrm{m}^{2}$ ), mientras en Junquillal fue la más baja (178 ind. $/ \mathrm{m}^{2}$ ), pese a que ambas playas son protegidas. Las playas no protegidas fueron menos variables, si se dan densidad bajas como en Playa Hermosa, Playa Matapalo y Sámara (Cuadro 3). Playas del Coco (1960 ind. $\left./ \mathrm{m}^{2}\right)$, Playa Tamarindo (847 ind. $/ \mathrm{m}^{2}$ ) y Playa Tambor (1622 ind. $/ \mathrm{m}^{2}$ ) presentaron densidad mayores (Cuadro 3), aunque las primeras dos fueron muestreadas hace varias decadas, por lo que su densidad actual no se conoce.

\section{DISCUSIÓN}

\section{Costa rocosa}

Los diferentes objetivos de los estudios sobre especies de invertebrados marinos de la zona intermareal rocosa realizados a lo largo de los años en las costas del Pacífico Norte de Costa Rica, influencian en gran medida la riqueza y abundancia de especies de invertebrados encontrados. Con esta información y en función del año en que se obtuvieron los datos, se puede detectar las tendencias a lo largo del tiempo (Fig. 2). Así, antes de 1970 se cuenta con esporádicas recolectas de poliquetos (Dean, 2009), durante la década de los setenta se inició también, una serie de muestreos de moluscos en los intermareales rocosos de Sámara, Herradura y playas del Coco (Miller, 1974; Spight 1976; 1977; 1978). Teniendo en cuenta las especies encontradas en los diferentes hábitats y años de muestreo, estos estudios reportaron de 15 a 91 especies por localidad. Después, a finales de los setenta se dieron (los) muestreos en Montezuma y Bahía Ballena (Playa Tambor), estudiando sólo la epifauna de la roca expuesta a la desecasión, que contó 
con un bajo número de especies (9-14), que corresponden a las más frecuentes (Villalobos, 1980a; b). Desde esa época y hasta, a inicios de los noventas, se dio en Guanacaste recolectas y anotaciones de invertebrados incrustantes, endolíticos y bioerosionadores (Fischer, 1981; Pepe, 1985; Dean, 2001b), si bien reportándose pocas especies. A partir del 2004, se retomó el tema de la epifauna y los valores variaron de 9 a 15 especies, similar en número e identidad de especies con los estudios de Villalobos (1980a; b); entre ellos está el trabajo de Sibaja-Cordero \& Vargas-Zamora (2006); Jörger, Meyer \& Wehrtmann (2008) y SibajaCordero \& Cortés (2008). El presente informe añade datos del 2007 de Playa Virador, en el Golfo de Papagayo (2007), que apunta a una diversidad similar de epifauna. La cantidad de especies se incrementó mucho al estudiar todos los componentes de la comunidad (epifauna, fauna incrustante, endolítica, bajo las rocas y en las algas marinas), como reflejan los datos del 2012, donde la riqueza de especies osciló entre 29 y 175 especies, según la localidad. Este número fue alto pese a que los datos fueron obtenidos en un único evento de muestreo en cada localidad. Estudios como los del Miller (1974) en Sámara y Hermosa; y los de Spigth (1976) que reportaron gran cantidad de especies, reflejan la importancia de hacer varios muestreos en la misma zona.

Los estudios realizados previamente en la región del Pacífico Norte presentan resultados concordantes con las observaciones realizadas en el presente estudio, para citar un ejemplo tenemos la similitud existente en la identificación de las especies que definen el paisaje de la zonación vertical, como las algas, Ulva y Halimeda; los cirripedios, C. panamensis y T. stalactifera; los moluscos, A. brevidentata, S. gigas o $S$. maura y litorínidos. En la zona de Bahía Salinas, Sibaja-Cordero \& Cortés, 2008, señalan coberturas altas de los mismos grupos sésiles, pero estos varían a lo largo del año dependiendo de la presencia o no de afloramiento, lo que condiciona la presencia y abundancia de las especies de moluscos móviles. Esta situación es de esperarse en mayor o menor medida para las playas muestreadas del Golfo de Santa Elena y Golfo de Papagayo; se sugiere realizar muestreos a largo plazo para así obtener un listado mas completo. En ese sentido, estudios temporales como los de Sipght (1978), brindaron una mayor riqueza de especies de moluscos, pero en muchos otros casos sólo se muestreó una parte de los grupos zoológicos y algales; y no a todas las especies presentes (Pepe, 1985; Sibaja-Cordero \& Cortés, 2008). En muchos de los lugares estudiados en el 2012 presentaron grupos de crustáceos como un componente importante de la riqueza del hábitat rocoso.

En algunos casos como la localidad de Tambor, se visitó la misma zona rocosa que muestreó Villalobos (1980b) observándose que los patrones de distribución y números relativos de especies resultaron similares; sin embargo, algunas de las especies encontradas por él, fueron halladas en el presente muestreo, pero se presentaron otros de moluscos, como las babosas marinas (Heterobranchia). En lo referente a poliquetos sésiles del género Sabellaria reportados por Villalobos (1980b) no fueron encontrados en el presente muestreo.

\section{Playas de Arena}

Para el Pacífico Norte de Costa Rica, esto, basándose en función del estudio realizado por Dexter (1974) y en los resultados del presente muestreo, podemos concluir que la riqueza de especies de las playas de arena del Pacífico Norte de Costa Rica es baja, si se compara con otros habitats marinos, presentando entre 2 y $<20$ especies por playa; sin embargo, Defeo \& McLachlan (2013) indican que organismos marinos en playas de arena alrededor del mundo, alcanzan como maximo de riqueza cerca de 30 especies. Así playas con valores de 20 , presentan una riqueza alta para este tipo de ambiente. Los datos de playas muestreadas en 2012 resultaron en una riqueza de especies entre 5 a 15. Playa Tambor en el presente muestreo, fue la playa arenosa que presentó el mayor número de especies, posiblemente al ser la más resguardada del efecto del oleaje que 
el resto de playas muestreadas. Dexter (1974) detalló la presencia de varias especies en playas como Tamarindo, El Coco y Sámara; donde el desarrollo de infraestructura (y visitación) turística ha crecido, y en dichas localidades, esto podría provocar una disminución en el número de especies presentes en la actualidad. Es de esperar que playas con poco desarrollo turístico (en la zona) cuenten con una riqueza de especies mayor.

\section{Efecto de las zonas protegidas}

Costa rocosa: Con base a los datos obtenidos para la costa rocosa, se encontró, que analizando los estudios previos junto con los actuales las zonas protegidas de manera general presentan un promedio mayor de especies que la zonas no protegidas (por alguna medida de conservación) aunque no es significativo debido al amplio ámbito de dispersión de los estimadores; mucho de ello, es debido al enfoque de los estudios realizados, donde no se tomaba en cuenta toda la comunidad. Al evaluar solo los datos del presente estudio en el 2012, se confirma que el promedio no es diferente y que se pueden encontrar valores altos tanto fuera como dentro de las zonas protegidas. En ese sentido, si se dieron zonas protegidas con alta riqueza de especies (ej. San Miguel en Cabo Blanco), pero otras cuentan con riqueza minima (El Hachal). Regiones como Sámara, Herradura o Matapalo sin protección o reguladas, fueron bastante ricas en especies.

Del parrafo anterior se entiende que no siempre las zonas protegidas están ubicadas donde existe una mayor diversidad de organismos en la zona intermareal y que, por lo tanto, muchos sitios con una gran variedad de invertebrados están todavía sin protección. La información de riqueza de especies en la costa rocosa debería tenerse en cuenta al planearse futuros esfuerzos de conservación.

También, de los listados de especies de moluscos obtenidos en el presente estudio, se encontró que todos los sitios visitados presentaban una riqueza de especies moderada, con excepción del Sector San Miguel en Cabo
Blanco, Reserva Absoluta donde no hay ingreso de pescadores o turistas. En ese sentido, el estudio de Casu, Ceccherelli, Curini-Galletti \& Castelli (2006), en Italia explica en parte el patrón encontrado en Costa Rica. Ellos notaron que en zonas protegidas italianas donde la visita de turistas está concentrada en los meses estivales, las actividades de trampeo sobre los organismos no eran significativas sobre su abundancia y diversidad; pero si se observó que dependiendo de la intensidad de captura de invertebrados por humanos, en un experimento, la presencia de estos y otros no capturados declinaba. Los autores resumen sus resultados indicando que al no haber un efecto constante del trampeo por turistas en las zonas protegidas, se evitaba una pérdida de riqueza de especies. Por otro lado, la extracción sostenida puede reducir dicha riqueza en mayor o menor medida, dependiendo de la intensidad de esa extracción. Micheli, Halpern, Botsford \& Warner (2004) indican que para el caso de peces, las zonas protegidas pueden incrementar la biomasa y la abundacia de las especies blanco de la extracción, si esto aplica para los invertebrados bentónicos, puede explicar el patrón hallado.

Una causa que puede explicar el amplio ámbito de riqueza encontrada entre los diferentes sitios, ya sean protegidos o no, puede deberse a la heterogeneidad del hábitat y a la presencia de perturbaciones en los sitios estudiados, ya sean estos naturales o antrópicos (Connell 1978). Por ejemplo, se encontró una baja riqueza de especies en El Hachal, como se indicó antes, pese a ser una zona protegida. Esto, se puede atribuir al tipo de substrato presente en la zona (cantos rodados), cuyas rocas presentan superficies muy lisas donde no crecen algas ni se forman grietas, por lo tanto no propician el establecimiento de invertebrados. Los cantos rodados poseen gran inestabilidad, resultando en una baja riqueza de especies; pues al voltearse por la fuerte acción del oleaje dichos cantos pueden dejar a los organismos que se asientan oculto, y expuestos a la desecasión a los que habitan debajo de estas; además, muchos organismos pueden sufrir daño 
mecánico (Little \& Kitching, 1996; Little, 2000). Otro ejemplo de perturbación ambiental es el caso de Cabuya dentro de Cabo Blanco, que, pese a ser una zona protegida, presentó una baja riqueza de especies y abundancia de moluscos marinos. Cabuya está siendo afectada por un fenómeno de erosión natural, que impide el crecimiento o establecimiento significativo de macroalgas, debido al efecto abrasivo del sedimento, o por depositarse sobre la roca, reduciendo la capacidad de forrajeo de muchos gastrópodos, generando dificultad para ciertos grupos filtradores (e.g. bivalvos y gusanos sabeláridos). Sin embargo, y al igual que ocurre en San Miguel, en Cabuya el sustrato es roca del tipo arenisca, lo que permite la presencia de especies horadadoras y la creación de espacios en la roca y aumentando la heterogeneidad del hábitat, que son utilizados por otros organismos, incluidos los poliquetos depredadores.

Finalmente, el intermareal rocoso de Matapalo presenta una alta diversidad de familias/ especies de moluscos y crustáceos; podría verse reducida por la presencia y ampliación de un complejo turistico en las cercanías, que poblaría la zona de visitantes, y junto con la presencia de pescadores artesanales recolectores de moluscos, introducirían presión sobre dicho hábitat.

La riqueza de especies dentro de una zona protegida puede aumentar o disminuir, dependiendo de que las especies extraídas o dañadas por actividades humanas se recuperen; pero puede ocurrir que una vez una zona presenta medidadas de conservación, al cabo de un tiempo ciertos depredadores o herbívoros se recuperan y reducen la cantidad de especies que son presas o competencia (Manríquez \& Castilla, 2001; Micheli et al., 2004). En este sentido, habría que analizars si los altos valores de riqueza de especies encontrados en zonas no protegidas, como Matapalo o playa Hermosa, se deben a la ausencia de ciertos depredadores clave, o si esta riqueza de especies es debida a la dominancia del alga Caulerpa sertularioides en el infralitoral, que ofrece un espacio tridimensional para el asentamiento y desarrollo de organismos asociados.

Playas de arena: También, como en la costa rocosa, en las playas de arena, no se encontró una mayor riqueza de especies dentro o fuera de las zonas protegidas, debido a la alta variabilidad de los datos. Lo mismo ocurre si tomamos en cuenta los datos de densidad, donde Playa Ventanas, presenta una densidad de 4267 individuos $/ \mathrm{m}^{2}$, mientras Junquillal solo 178 ind. $/ \mathrm{m}^{2}$, ambas playas protegidas. La alta densidad en Ventanas es producto de la gran cantidad de isopodos cirolánidos en el litoral alto y supralitoral. La densidad promedio de las playas no protegidas del Cuadro 3 fue de $910 \mathrm{ind} . / \mathrm{m}^{2}$, donde sitios como las playas Hermosa y Matapalo, muestreadas en 2012 presentaron una densidad baja y que como se mencionó antes presentaban infraestructura aledana a la playa y alta cantidad de turistas. Otros sitios en igual situación en la actualidad en los datos del Cuadro 3, pertenecientes al estudio de Dexter (1974), presentaban una densidad alta (como Playas del Coco y Playa Tamarindo) o ya baja (como Playa Sámara); por lo que se hace necesario un nuevo muestreo de estas para poder comparar datos y determinar cuanto han cambiado dichas playas con el desarrollo de asentamiento humanos. Finalmente Playa Tambor, que es más resguardada del oleaje al estar en Bahía Ballena, presentó una densidad alta de organismos (1622 ind. $\left./ \mathrm{m}^{2}\right)$. De esta manera, es claro que muchas playas de arena como Tambor, El Coco, Sámara y Carillo ubicadas dentro de bahías, más resguardadas y más disipativas, de grano más fino, es donde se puede esperar una mayor riqueza de especies y mayor densidad de individuos, que en playas reflectivas y expuestas al oleaje, de grano más grueso, como mencionan Defeo \& McLachlan (2005), para este ambiente marino. Pero también, se nota en los datos que la presencia de asentamiento humanos puede reducir la densidad de fauna presente en las playas del Pacifico Norte de Costa Rica. 


\section{Conclusiones y recomendaciones}

Las zonas rocosas del presente estudio y que fueron muestreadas en el año 2012 presentaron riqueza de especies superiores (29 a 172) a las aportadas por muchos de los trabajos realizados anteriormente en esta zona del Pacífico costarricense, que se basaban en ciertos grupos taxonómicos o ecológicos y no en la totalidad de la comunidad. Esto establece la importancia de dichos ambientes en cuanto a riqueza de especies en la costa del país.. Estas listas podrían incluso verse incrementadas si se realizaran estudios temporales de la composición de las comunidades bentónicas. Las playas de arena presentan desde baja a media riqueza de macrofauna (5-15), al comparar con datos a nivel mundial; sin embargo, la fauna de dicha zona está muy adaptada a estos ambientes y es importante su conservación. Los resultados generales apuntan a que hay muchas playas y zonas rocosas de la costa del Pacífico Norte de Costa Rica que no están protegidas y que muestran una riqueza de especies igual o incluso superior a las de las zonas protegidas de la región, y es sobre estas localidades donde debería prestarse una atención particular para su conservación futura. Concretamente, entre las recomendaciones planteadas están:

1. Hacer un monitoreo en los sitios no protegidos que presentan una alta riqueza de especies, tales como Playa Matapalo, Hermosa, Sámara y las playas del Golfo de Santa Elena.

2. Conservar las costas del Golfo de Santa Elena debido a la alta riqueza de especies observada en los diferentes sitios visitados. También, cabe resaltar que la belleza paisajística/escénica debe ser preservada, no permitiendo el desarrollo de complejos turísticos en la zona.

3. Buscar alternativas de mitigación para las zonas protegidas amenazadas por fenómenos naturales como en el caso de Cabuya en La Reserva Natural Absoluta de Cabo Blanco.
4. Instar a las empresas hoteleras que construyen grandes complejos turísticos cerca de zonas rocosas extensas, con alta riqueza de especies, a que contribuyan a la protección de las mismas, implementado programas de educación ambiental entre su personal y visitantes turísticos.

5. Monitorear los mismos lugares permitiendo así la comparación de los datos y visualizar las zonas donde no se han hecho estudios de los invertebrados de la costa de Guanacaste, zona vulnerable por el desarrollo turístico.

\section{AGRADECIMIENTOS}

Se agradece al proyecto 808-B2-400 financiado por la Agencia Alemana de Cooperación GiZ, (Proyecto BIOMARC), la Asociación Costa Rica por Siempre, la Vicerrectoría de Investigación y CIMAR de la Universidad de Costa Rica. A Carlos Garita, Kimberly García, Marco Corrales y Carolina Salas por su colaboración en el muestreo y en el trabajo de laboratorio. A los revisores anónimos, a Omar Defeo de la Universidad de la República, Uruguay y Juan Moreira de la Universidad Autónoma de Madrid por sus comentarios.

\section{RESUMEN}

Este estudio resume información publicada sobre las especies de invertebrados reportadas para la zona intermareal de la costa rocosa y las playas de arena del Pacífico Norte de Costa Rica. Además, se presentan datos nuevos sobre la riqueza de especies de la comunidad de invertebrados de varias playas de áreas protegidas o no protegidas muestreadas durante el año 2012. Los resultados indican una amplia variación en el número de especies reportadas, siendo los principales contribuyentes a la fauna los moluscos, crustáceos y poliquetos. El número de especies dependió del grupo faunístico y el esfuerzo de muestreo de los estudios previamente realizados. Teniendo en cuenta, todos los componentes de la comunidad, se encontraron entre 29 a 175 especies en los intermareales rocosos, y de 5 a 15 especies en las playas de arena. El valor promedio de riqueza de especies fue similar entre las áreas protegidas y no protegidas, aunque en ambos casos el rango de valores fue muy amplio. Hay lugares con gran riqueza de especies, sin medidas de protección, que están actualmente 
expuestos a los impactos del desarrollo de las poblaciones humanas, y es ahí donde urge la gestión de dichos hábitats.

Palabras clave: Riqueza de especies, playas tropicales, crustáceos, moluscos, poliquetos, algas.

\section{BIBLIOGRAFÍA}

Böggemann, M. (2002). Revision of the Glyceridae Grube 1850 (Annelida, Polychaeta). Abhandlungen der Senckenbergischen Naturforschenden Gesellschaft, $555,1-249$.

Camacho-García, Y., Gosliner, T., \& Valdés, A. (2005). Field guide to the sea slugs of the Tropical Eastern Pacific. California: California Academy of Sciences.

Casu, D., Ceccherelli, G., Curini-Galletti, M., \& Castelli, A. (2006). Human exclusion from rocky shores in a mediterranean marine protected area (MPA). An opportunity to investigate the effects of trampling. Marine Environmental Research, 62, 15-32.

Connell, J. H. (1978). Diversity in tropical rain forests and coral reefs. Science, 199, 1302-1310.

Dean, H. K. (2001a). Some Nereididae (Annelida, Polychaeta) from the Pacific Coast of Costa Rica. Revista de Biología Tropical, 49 (Supplement 2), 37-67.

Dean, H. K. (2001b). Marine biodiversity of Costa Rica, the phyla Sipuncula and Echiura. Revista de Biología Tropical, 49 (Supplement 2), 85-90.

Dean, H. K. (2004). Marine biodiversity of Costa Rica, Class Polychaeta (Annelida). Revista de Biología Tropical, 52(Supplement 2), 131-181

Dean, H. K. (2009). Polychaetes and echiurans. In, I. S. Wehrtmann, \& J. Cortés (Eds.), Marine Biodiversity of Costa Rica, Central America. (Text: pp. 181-191, Species List: 122-159pp). Berlin: Springer + Business Media B. V.

Defeo, O. \& McLachlan, A. (2005). Patterns, processes and regulatory mechanisms in sandy beach macrofauna: a multi-scale analysis. Marine Ecology Progress Series, 295, 1-20.

Defeo, O. \& McLachlan, A. (2013). Global patterns in sandy beach macrofauna: species richness, abundance, biomass and body size. Geomorphology, 199, 106-114.

Defeo, O., McLachlan, A., Schoeman, D. S., Schlacher, T. A., Dugan, J., Jones, A., Lastra, M., \& Scapini, F. (2009). Threats to sandy beach ecosystems, a review. Estuarine, Coastal and Shelf Science, 81, 1-12.

de León, J. A., Bastida, J. R., Carrera, L. F., García, M. E., Peña, A., Salazar, S. I., \& Solís, S. (2009). Poliquetos (Annelida, Polychaeta) de México y América
Tropical. Monterrey: Universidad Autónoma de Nuevo León.

Dexter, D. M. (1974). Sandy-beach fauna of the Pacific and Atlantic coasts of Costa Rica and Colombia. Revista de Biología Tropical, 22, 51-66.

Fauchald, K. (1973). Polychaetes from Central American sandy beaches. Bulletin, Southern California Academy of Science, 72, 19-31.

Fischer, R (1981). Bioerosion of basalt of the Pacific coast of Costa Rica. Senckenbergiana maritima, 13, 1-41.

Fischer, W., Krupp, F., Schneider, W., Sommer, C., Carpenter, K. E., \& Niem, U. H. (eds.) (1995). Guía FAO para la identificación de especies para los fines de la pesca. Pacífico Centro-oriental. Vol. 1. Plantas e Invertebrados. Rome: FAO.

Garth, J. S. (1958). Brachyura of the Pacific coast of America. Oxyrhyncha. Allan Hancock Pacific Expedition, $21,1-499$.

Haig, J. (1960). The Porcellanidae (Crustacea, Anomura) of the eastern Pacific. Allan Hancock Pacific Expedition, 24, 1-440.

Hendrickx, M. E. (1999). Los cangrejos braquiuros del Pacífico mexicano (Crustacea, Brachyura, Majoidea y Parthenopoidea). México: Comisión Nacional para el Conocimiento y Uso de la Biodiversidad e Instituto de Ciencias del Mar y Limnología, UNAM.

Jörger, K., Meyer, R., \& Wehrtmann, I. S. (2008). Species composition and vertical distribution of chitons (Mollusca, Polyplacophora) in a rocky intertidal zone of the Pacific coast of Costa Rica. Journal of the Marine Biological Association of the United Kingdom, 88, 807-816

Keen, A. M. (1971). Sea Shells of Tropical West America. Marine Mollusks from Baja California to Peru, (2nd ed), California: Stanford.

Kim, W., \& Abele, L. G. (1988). The snapping shrimp genus Alpheus from the Eastern Pacific (Decapoda, Caridea, Alpheidae). Smithsonian Contributions to Zoology, 454, 1-119.

Laguna, J. E. (1985). Systematics, Ecology and Distribution of Barnacles (Cirripedia; Thoracica) of Panama, Including an Analysis of Pprovincialism in the Tropical Eastern Pacific. M.Sc. Thesis. San Diego, California: University of California.

Little, C. \& J. A. Kitching. (1996). The Biology of Rocky Shores. Oxford: Oxford University Press.

Little, C. (2000). The Biology of Soft Shores and Estuaries. Oxford: Oxford University Press.

Madrigal-Castro, E., Cabrera-Peña, J., Monge-Esquivel, J. \& Pérez-Acuña, F. (1984). Comparación entre dos poblaciones de Acanthina brevidentata (Gastropoda, Mollusca) en dos zonas rocosas de Playa Panamá, 
Guanacaste, Costa Rica. Revista de Biología Tropical, 32, 11-15.

Manríquez, P. H. \& Castilla, J. C. (2001). Significance of marine protected areas in central Chile as seeding grounds for the gastropod Concholepas concholepas. Marine Ecology Progress Series, 216, 201-211.

Micheli, F, Halpern, B. S., Botsford, L. W. \& Warner, R. R. (2004). Trajectories and correlates of community change in no-take marine reserves. Ecological Applications 14(6), 1709-1723.

Miller, A. C. (1974). A Comparison of Gastropod Diversity and Trophic Structure in the Rocky Intertidal Zone of the Temperate and Tropical West America. Ph.D. Thesis. Eugene, Oregon: University of Oregon.

Pepe, P. J. (1985). Littoral endolithic fauna of the Central American Isthmus. Revista de Biología Tropical, 33, 191-194.

Rathbun, M. J. (1930). The cancroid crabs of America of the families Euryalidae, Portunidae, Ateleeyelidae, Cancridae and Xanthidae. United States National Museum-Bulletin, 152, I--XVI, 1--609.

Salgado-Barragán, J., \& Hendrickx, M. E. (2010). Clave ilustrada para la identificación de los estomatópodos (Crustacea, Hoplocarida) del Pacífico Oriental. Revista Mexicana de Biodiversidad, 81 (Supplement), 1-49.

Sibaja-Cordero, J. A., \& Vargas-Zamora, J. A. (2006). The vertical zonation of epifauna and algae species in rocky substrates of the Gulf of Nicoya, Costa Rica. Revista de Biología Tropical, 54(Supplement 1), 49-67.
Sibaja-Cordero, J. A. \& Cortés, J. (2008). Vertical zonation of rocky intertidal organisms in a seasonal upwelling area (Eastern Tropical Pacific). Revista de Biología Tropical, 56, 91-104.

Spight, T. M. (1976). Censuses of rocky shore prosobranchs from Washington and Costa Rica. Veliger, 18, 309-317.

Spight, T. M. (1977). Diversity of shallow-water gastropod communities on temperate and tropical beaches. American Naturalist, 111, 1077-1097.

Spight, T. M. (1978). Temporal changes in a tropical rocky shore snail community. Veliger, 21, 137-143.

Vargas, J. A. (1987). The benthic community of an intertidal mud flat in the Gulf of Nicoya, Costa Rica. Description of the community. Revista de Biología Tropical, 35, 299-316.

Vargas, J. A., \& Dean, H. K. (2009). Sipunculans. In, I. S. Wehrtmann, \& Cortés, J. (eds.), Marine Biodiversity of Costa Rica, Central America. (Text: pp. 119-121). Berlin: Springer + Business Media.

Villalobos, C. R. (1980a). Variations in population structure in the genus Tetraclita (Crustacea, Cirripedia) between temperate and tropical populations. III. T. stalactifera in Costa Rica. Revista de Biología Tropical, 28, 193-201.

Villalobos, C. R. (1980b). Algunas consideraciones sobre el efecto de los factores físicos y biológicos en la estructura de una comunidad de algas en el Pacífico de Costa Rica. Brenesia, 18, 289-300. 